\title{
Review Article \\ Hypoxia-Inducible Factor in Thyroid Carcinoma
}

\author{
Natalie Burrows, ${ }^{1}$ Muhammad Babur, ${ }^{1}$ Julia Resch, ${ }^{2}$ Kaye J. Williams, ${ }^{1}$ and Georg Brabant ${ }^{2}$ \\ ${ }^{1}$ Hypoxia and Therapeutics Group, School of Pharmacy and Pharmaceutical Sciences, University of Manchester, Oxford Road, \\ Manchester M13 9PT, UK \\ ${ }^{2}$ Experimental and Clinical Endocrinology, Medizinische Klinik I Ratzeburger Allee 160, 23538 Lübeck, Germany
}

Correspondence should be addressed to Kaye J. Williams, kaye.williams@manchester.ac.uk

Received 7 March 2011; Accepted 20 April 2011

Academic Editor: Cuong Hoang-Vu

Copyright () 2011 Natalie Burrows et al. This is an open access article distributed under the Creative Commons Attribution License, which permits unrestricted use, distribution, and reproduction in any medium, provided the original work is properly cited.

\begin{abstract}
Intratumoural hypoxia (low oxygen tension) is associated with aggressive disease and poor prognosis. Hypoxia-inducible factor-1 is a transcription factor activated by hypoxia that regulates the expression of genes that promote tumour cell survival, progression, metastasis, and resistance to chemo/radiotherapy. In addition to hypoxia, HIF-1 can be activated by growth factor-signalling pathways such as the mitogen-activated protein kinases- (MAPK-) and phosphatidylinositol-3-OH kinases- (PI3K-) signalling cascades. Mutations in these pathways are common in thyroid carcinoma and lead to enhanced HIF-1 expression and activity. Here, we summarise current data that highlights the potential role of both hypoxia and MAPK/PI3K-induced HIF-1 signalling in thyroid carcinoma progression, metastatic characteristics, and the potential role of HIF-1 in thyroid carcinoma response to radiotherapy. Direct or indirect targeting of HIF-1 using an MAPK or PI3K inhibitor in combination with radiotherapy may be a new potential therapeutic target to improve the therapeutic response of thyroid carcinoma to radiotherapy and reduce metastatic burden.
\end{abstract}

\section{Introduction}

The hypoxia-inducible factors (HIFs) are transcription factors that function under low oxygen tensions (hypoxia) and are, therefore, active in a number of diseases associated with low oxygen $\left(\mathrm{O}_{2}\right)$ environments. These include ischemic disorders, atherosclerosis, and importantly cancer. HIF drives the survival and development of cancer cells by activating and repressing a multitude of genes that promote tumour cell survival, proliferation, invasion, and disease progression. As a result, hypoxia and HIF are associated with poor prognosis in many tumour types [1-3]. Hypoxia occurs in the majority of solid tumours, thus functional HIF is present in most tumour types indicating the importance of this signalling pathway in cancer. There is little known, however, about the role of HIF in thyroid carcinoma. Here, we summarise current literature that supports the potential significance of the HIF signalling pathway in progression and aggressiveness of thyroid carcinoma. Current data proposes that the HIF pathway may be a novel therapeutic target in reducing local tumour growth, metastatic burden, and resistance to chemo/radiotherapy.

\section{Oxygen-Dependent Regulation of HIF-1}

There are three known isoforms of HIF: HIF-1, 2, and 3. HIF-1 is expressed in all cells and is the most extensively researched, whereas the expression of the other isoforms is restricted to certain tissues. HIF-1 is a heterodimeric protein consisting of a constitutively expressed HIF-1 $\beta$ (also known as the ary lhydrocarbon receptor nuclear translocator; ARNT) subunit and an oxygen-labile HIF$1 \alpha$ subunit. Under conditions of low oxygen, HIF- $1 \alpha$ is stabilised, heterodimerises with HIF- $1 \beta$ through the PerARNT-Sim (PAS) A and PAS B domains, and translocates to the nucleus. The complex then binds to the hypoxiaresponsive element (HRE; consensus sequence G/ACGTG), in the promoter region of target genes via the basic helixloop-helix (bHLH) DNA-binding domain and activates transcription. This process involves binding of the coactivators CREB-binding protein (CBP) and p300 [4, 5]. 
Under normoxia, HIF- $1 \alpha$ is hydroxylated on proline residues 402 and/or 564 in the oxygen-dependent degradation domain (ODD). This process is carried out by specific oxygen-dependent enzymes known as proline hydroxylase domain proteins (PHDs). There are 3 PHDs: 1, 2, and 3. PHD2 is specifically involved in the hydroxylation of HIF- $1 \alpha$. The PHDs use $\mathrm{O}_{2}$ and 2-oxoglutarate (2-OG) as substrates. Upon hydroxylation, von Hippel-Lindau (VHL), a tumoursuppressor protein, binds HIF- $1 \alpha$ and recruits the E3 ubiquitin ligase, leading to ubiquitination and proteosomal degradation of HIF- $1 \alpha$ (Figure 1). Activation of asparaginyl hydroxylases such as factor inhibiting HIF-1 (FIH-1) represents an additional oxygen-dependent mechanism of inhibition of HIF- $1 \alpha$ activity. FIH-1 hydroxylates asparagine803 in the C-terminal transcriptional activation domain (CTAD) of HIF- $1 \alpha$. This modification inhibits the interaction of C-TAD with the transcriptional co-activators $\mathrm{CBP} / \mathrm{p} 300$, and thus inhibits the transcriptional activity of HIF-1 (Figure 1). Under hypoxia, the level of HIF- $1 \alpha$ hydroxylation is reduced via inhibition of the PHD2 enzyme, resulting in stabilisation and accumulation of HIF- $1 \alpha$ protein $[4,5]$. An additional oxygen-sensitive mechanism of HIF-1 regulation is the generation of reactive oxygen species (ROS) from mitochondria. ROS inactivate PHD2 resulting in direct stabilisation of HIF- $1 \alpha[6]$.

HIF- $2 \alpha$ is likewise regulated by oxygen-dependent hydroxylation and dimerises with HIF- $1 \beta$ to form the functional HIF-2 complex $[4,7]$. Both isoforms are similar in structure and function but have differences, particularly in the N-TADs [8]. This suggests that both isoforms may differ in the activation of target genes and the recruitment of coactivators. There are also fewer HIF- $2 \alpha$-regulated genes compared to HIF- $1 \alpha$. For example, in MCF-7 breast carcinoma cells, $80 \%$ of hypoxia-regulated genes were dependent on HIF- $1 \alpha$. A small group were dependent on HIF- $2 \alpha$, and the regulation of these genes was due to the interaction of HIF- $2 \alpha$ with the transcription factor Elk-1 [9]. This interaction with Elk-1 is unique to HIF- $2 \alpha$.

Although HIF- $1 \alpha$ and $-2 \alpha$ show some overlap of target genes, the proteins do have distinct downstream targets. HIF1 predominantly regulates the expression of genes encoding glycolytic proteins such as lactate dehydrogenase-A (LDHA) and carbonic anhydrase-9 (CA-9), whereas in certain tissues expressing both HIF- 1 and $-2 \alpha$, expression of genes such as vascular endothelial growth factor (VEGF) and erythropoietin (EPO) is mainly regulated by HIF- $2 \alpha[7$, $10,11]$. In support of this, high expression of HIF- $2 \alpha$ but not $-1 \alpha$ has been found in well-vascularised areas of neuroblastoma and is associated with aggressiveness [12]. Although HIF- $1 \alpha$ is accepted as the most important of the HIFs, there is increasing evidence suggesting that HIF- $2 \alpha$ may be of equal significance. The expression of HIF- $2 \alpha$ is both tissue and cell-type specific, and the regulation of target genes differs depending on tissue type, tumour type, and coexpression with HIF- $1 \alpha$. A better understanding of how these factors lead to cell-specific differences in HIFdependent gene regulation may help in the development of more effective therapeutics for diseases highly dependent on hypoxia. The HIF- $3 \alpha$ isoform is also hypoxia regulated in a HIF-1-dependent manner and is an inhibitor of HIF-1 function [13].

\section{Oxygen Independent Mechanisms of HIF Activation}

3.1. Mutations in VHL. VHL targets all the HIF- $\alpha$ s for rapid proteosomal degradation and, as a result, plays a central role in molecular oxygen sensing [14]. Studies into the phenotype of VHL knockout (KO) mice provide direct evidence for the physiological relevance of the HIF pathway and VHLregulated expression of HIF- $1 \alpha$ in both normal foetal development and cancer progression. Genetic knock-out of VHL in the murine germ line results in embryonic lethality in midgestation due to abnormal vasculature formation thought to be HIF dependent [15]. In certain hereditary cancers such as clear cell renal carcinomas, Chuvash polycythemia, pheochromocytoma of the central nervous system or hemangioblastoma of the retina, normoxic degradation of HIF are impaired due to mutations in VHL. These tumours are usually well vascularised, a characteristic largely attributed to deregulated HIF- $1 \alpha$ signalling $[16,17]$.

3.2. Metabolic Signalling Pathways. Metabolic signalling pathways such as the tricarboxylic acid (TCA) cycle are also necessary for normal regulation of HIF- $1 \alpha$. In addition to $\mathrm{O}_{2}$, PHDs require the substrates 2-OG and ascorbic acid as a cofactor, to catalyze hydroxylation of HIF- $1 \alpha$. This reaction produces succinate and $\mathrm{CO}_{2}$ as byproducts. 2-OG is a metabolite of the TCA cycle. As 2-OG is required for the functionality of PHDs, activity of the TCA cycle regulates PHD activity and thus HIF- $1 \alpha$ stability. Furthermore, inactivating mutations in enzymes of the TCA cycle leads to direct stabilisation of HIF- $1 \alpha$ protein $[18,19]$. Mutations in fumarate hydratase $(\mathrm{FH})$ and succinate dehydrogenase (SDH) lead to accumulation of fumarate and succinate. Both fumarate and succinate inhibit PHDs by competing with 2-OG for binding to the active site [20,21]. Inactivating mutations in SDH have been found in a range of cancers associated with enhanced HIF-1 and VEGF signalling. These include renal cell carcinomas, gastrointestinal stromal (GIST) tumours, and Carney's syndrome [22, 23].

3.3. Growth Factor Signalling Pathways. For thyroid cancer the most important mutational changes occur in the MAPK/Ras - extracellular signal-regulated kinase (ERK) and the PI3K/AKT signalling cascades [24]. These pathways are dominant regulators of many cellular processes including growth, metabolism, cell survival, and angiogenesis and are important in the coordinated interaction of cells with the microenvironment $[25,26]$. Both the MAPK and PI3K signalling pathways increase HIF-1 signalling in many cancer types including thyroid [27-30].

3.4. HIF-1 and the MAPK Pathway. A number of MAPK (p38 and $\mathrm{p} 38 \gamma$ ) and ERK1/2 (p42 and p44) isoforms have been found to regulate HIF- $1 \alpha$ activity in a cell-specific manner. MAPK signalling enhances the transactivation of 


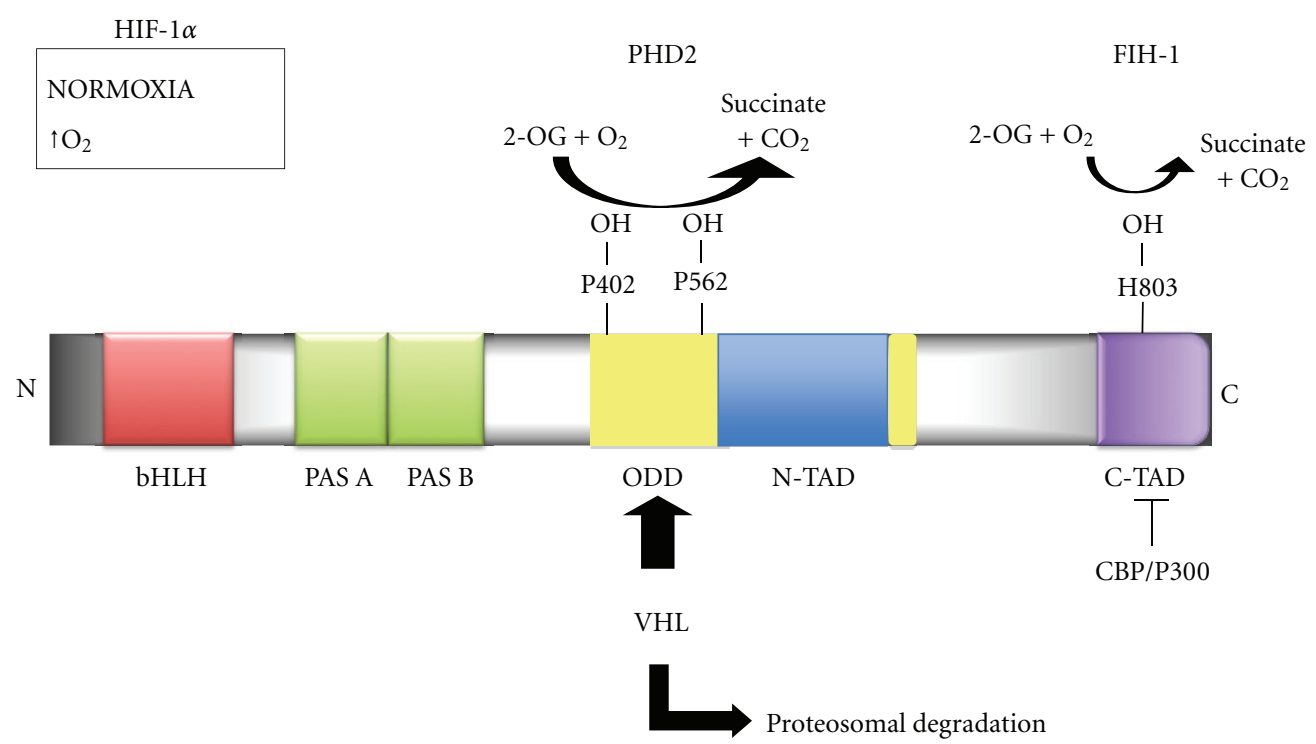

FIGURE 1: Structure of HIF- $1 \alpha$ and the oxygen-dependent regulation of HIF- $1 \alpha$ protein stabilisation and activation: The N-terminal regions contain the basic helix-loop-helix (bHLH) domain involved in DNA-binding and the Per-Arnt-Sim (PAS) A and B domains required for heterodimerisation with HIF-1 $\beta$. The oxygen-dependent degradation domain (ODD) is where PHD2 hydroxylates proline residues P402 and P562, which enables binding of VHL and proteosomal degradation. The terminal transcriptional activation domains (TADs) are responsible for transactivation of target genes. The N-TAD is located towards the N-terminus, with the C-TAD located at the extreme C-terminus. Factor inhibiting HIF-1 (FIH-1) hydroxylates asparagine H803 in the C-TAD, preventing the binding of coactivators CBP/p300, thus inhibiting activation of HIF- $1 \alpha$. Both hydroxylation processes use 2-oxoglutarate (2-OG) and $\mathrm{O}_{2}$ as substrates and produce succinate and carbon dioxide $\left(\mathrm{CO}_{2}\right)$ as byproducts.

HIF- $1 \alpha$ by phosphorylation of HIF- $1 \alpha$ co-activators and by direct phosphorylation of HIF- $1 \alpha$ itself. MAPK stimulation leads to the phosphorylation of $\mathrm{p} 300$. This phosphorylation facilitates binding of $\mathrm{p} 300 / \mathrm{CBP}$ to the C-TAD and promotes the transactivation activity of both $\mathrm{p} 300 / \mathrm{CBP}$ and HIF- $1 \alpha$. Studies suggest that MAPK does not phosphorylate FIH, and therefore, this signalling cascade is not involved in the oxygen-dependent regulation of HIF-1 $\alpha$ [31]. Thus, even though MAPK signalling does not depend on sensing oxygen tension, activation of this signalling cascade augments the HIF response.

Studies have shown that MAPK can directly phosphorylate the TADs of HIF- $1 \alpha$. Sang et al. found that MAPK signalling leads to indirect phosphorylation of CTAD constructs by MAPK and direct phosphorylation of $\mathrm{N}$ TAD constructs, when expressed in Escherichia coli. However, this direct phosphorylation of the TADs was not necessary for binding of p300 and activation of HIF-1 [31]. Other studies suggest that direct phosphorylation of HIF- $1 \alpha$ by MAPKs has functional consequences on HIF-1 activity. In HeLa and CCL39 cells, p42/44 MAPK (but not p38 MAPK and JNK) increased the transcriptional activity of HIF-1 by phosphorylation of HIF- $1 \alpha$ and not by increasing the level of HIF- $1 \alpha$ protein. This was blocked by the MEK inhibitor PD098059 [32]. Conversely, in Hep3B and HEK293 cells, activation of the small GTPase Racl by hypoxia leads to Rac1-dependent p38 MAPK signalling resulting in both enhanced phosphorylation of the N-TAD and transcriptional activity of HIF- $1 \alpha[33,34]$. Another study, however, has shown that Rac1 reduces HIF-1 activity by activating
NAPDH oxidases resulting in enhanced generation of ROS leading to reduced HIF-1 in both normoxia and hypoxia [35]. The effect of Rac1 on MAPK signalling was not shown in this study. Furthermore, the latter group used $8 \% \mathrm{O}_{2}$ for hypoxia, whereas the first study used 5 and $1 \%$ $\mathrm{O}_{2}$ for hypoxia. These functional differences observed by Racl may, therefore, be dependent upon $\mathrm{O}_{2}$ concentration. Furthermore, different cell lines were used in these studies suggesting that these differences in Rac1 signalling may be dependent on the activating mutation status of the MAPK pathway in different cell lines. Studies in HeLa cells have also shown that direct phosphorylation of HIF- $1 \alpha$ by p $42 / 44$ MAPKs not only leads to enhanced HIF-1 transcriptional activity, but also promotes nuclear accumulation of HIF- $1 \alpha$ [36]. Thus, depending on the cell type, the MAPK signalling cascade can enhance HIF-1 activity via activation of selective kinases which either directly phosphorylate HIF- $1 \alpha$ or coactivators of HIF- $1 \alpha$ (Figure 2).

In addition to MAPK signalling leading to enhanced activity of HIF-1, studies have also shown that the MAPK pathway may increase HIF- $1 \alpha$ protein synthesis in certain cancer types. Fukuda et al. showed that MAPK signalling can increase HIF- $1 \alpha$ protein synthesis in HCT116 colon carcinoma cells. Inhibitors of MAPK blocked HIF- $1 \alpha$ protein synthesis, and the overexpression of a constitutively active MAPK kinase (MEK2) induced HIF-1 $\alpha$ protein [37] (Figure 2).

Genetic mutations in the v-raf murine sarcoma viral oncogene homolog B1 (BRAF) gene, which encodes a serinethreonine kinase, result in hyperactive MAPK signalling. 
$\mathrm{BRAF}^{\mathrm{V} 600 \mathrm{E}}$ is the most common genetic mutation within papillary thyroid carcinomas (PTCs). On average, approximately $50 \%$ of all PTCs harbour the $\mathrm{BRAF}^{\mathrm{V} 600 \mathrm{E}}$ mutation [38]. This BRAF mutant is constitutively active and phosphorylates MEK1/2 leading to hyperactive MAPK signalling (Figure 2). It is thought that BRAF and, thus, MAPK signalling are important in the early stages of thyroid tumour development and predispose cells to become dedifferentiated tumours [39-41]. This has recently been substantiated in a mouse model of a thyroid-specific knock-in of oncogenic BRAF (LSL-BRAFV600E/TPO-Cre), which leads to a high frequency of invasive PTCs. These mice had high levels of thyroid stimulating hormone that acted cooperatively with oncogenic BRAF to drive tumour initiation [42].

The GTPase V-Ki-ras2 Kirsten rat sarcoma viral oncogene homolog (KRAS) is one of three members of the RAS family of GTPases. KRAS interacts with and activates a number of effector proteins including BRAF and PI3K. Genetic mutations in RAS leading to constitutive activity results in hyperactivation of the PI3K/MAPK pathways, promoting tumour progression in many cancer types including thyroid [43]. Recently, it has been shown that mutations in KRAS or BRAF differentially regulate HIF- $1 \alpha$ and HIF- $2 \alpha$ in colon carcinoma cells. Mutant KRAS enhanced expression of HIF$1 \alpha$ only, whereas mutant BRAF enhanced expression of both HIF- $1 \alpha$ and $-2 \alpha$. KRAS-induced HIF- $1 \alpha$ was dependent on PI3K activation, whereas only HIF- $2 \alpha$ was inhibited by the MEK inhibitor PD089059 in BRAF mutant cells [44]. These data highlight the complexity of HIF induction by oncogenic signalling cascades and may contribute to the phenotypic differences observed not only in colon cancer but other cancers harbouring these mutations such as thyroid.

The interplay and activation of MAPK by additional signalling pathways promotes HIF-1 activity. In colon cancer cells, loss of the tumour suppressor SMAD4 (mothers against decapentaplegic (MAD) and the Caenorhabditis elegans protein SMA-4) led to enhanced HIF- $1 \alpha$ activity through the activation of MEK-ERK and p38 MAPK by Transforming growth factor- $\beta$ (TGF- $\beta$ ) [45].

Stimulation of the MAPK cascade enhances HIF-1 signalling mainly by increasing the transcriptional activity of HIF- $1 \alpha$. This will lead to increased expression of HIF-1 downstream targets under normoxic conditions and in the presence of hypoxia will lead to a cooperative enhancement of expression of HIF-1 target genes.

3.5. HIF-1 and the PI3K Pathway. Aberrant activation of the PI3K pathway occurs by (a) hyperactive stimulation of receptor tyrosine kinases (RTKs) including the insulin-like growth factor-1 receptor (IGF-IR), the human epidermal growth factor receptor 2 (HER ${ }^{2 \text { neu }} \mathrm{R}$ ), and the epidermal growth factor receptor (EGFR) and (b) by mutational events that occur in negative regulators of PI3K signalling such as those in Phosphatase and tensin homolog (PTEN) and p53. Activation of the PI3K pathway is pivotal in the development and progression of thyroid cancer aggressiveness and appears to be predominantly involved in the metastatic behaviour of these tumours [41]. Hyperactive PI3K signalling leads to stabilisation of HIF- $1 \alpha$ in normoxia. This depends on the activation of AKT and subsequently mammalian target of rapamycin (mTOR), which increases the translation and activity of HIF- $1 \alpha[37,41,46,47]$.

mTOR is a serine/threonine kinase consisting of multiple protein-binding motifs that enable interaction and phosphorylation of many proteins and is considered a central signalling molecule for convergence and crosstalk between multiple pathways. mTOR phosphorylates and activates the translational machinery proteins: eukaryotic translation initiation factor 4E- (eIF-4E-) binding protein (4E-BP1) and p70 S6 kinase. Upon phosphorylation, 4E$\mathrm{BP} 1$ no longer interacts with and represses eIF- $4 \mathrm{E}$, and $\mathrm{p} 70^{\mathrm{S} 6}$ phosphorylates and activates the $40 \mathrm{~S}$ ribosomal protein. Activation of both eIF- $4 \mathrm{E}$ and the $40 \mathrm{~S}$ ribosomal protein initiates translation of HIF- $1 \alpha$ mRNA (Figure 3) $[48,49]$.

Stimulation of PI3K signalling by activation of different RTKs in a variety of cancer types induces protein synthesis of HIF- $1 \alpha$. This has been shown in colon and breast cancer cells via activation of IGF-1R and HER2 ${ }^{\text {nue }} R$ by IGF-1 and heregulin, respectively, [37, 50]. Stimulation of $\mathrm{PI} 3 \mathrm{~K} / \mathrm{AKT} / \mathrm{mTOR}$ induces the activation of the translational machinery proteins and protein synthesis of HIF$1 \alpha$. HER2 $2^{\text {neu }}$ is an indicator for poor prognosis in breast cancer and tumours overexpressing HER2 have increased angiogenesis due to increased expression of the HIF-1 target gene VEGF. Similarly, overexpression of HIF- $1 \alpha$ and VEGF has been found in prostate cancer cells that have hyperactive $\mathrm{PI} 3 \mathrm{~K} / \mathrm{AKT} / \mathrm{mTOR}$ activity as a result of enhanced EGFR signalling [51].

Depending on the type of RTK that is activated and on the type of cancer, increased HIF- $1 \alpha$ protein may be PI3K dependent even in the presence of activated MAPK signalling or may be dependent on both signalling pathways. Oestrogen-dependent tumours usually display hyperactive, PI3K, HIF- $1 \alpha$, and VEGF signalling. Studies on the effect of oestrogen induced HIF- $1 \alpha$ and VEGF in rat uterine tissue have shown that although oestrogen receptor signalling stimulates both the PI3K and MAPK signalling cascades, induction of HIF- $1 \alpha$ and VEGF was only dependent upon PI3K signalling [52]. However, in HCT116 colon cancer cells, IGF-1 induced HIF- $1 \alpha$ protein synthesis involved both the PI3K and MAPK signaling pathways [37]. Enhanced activity of HIF-1 by growth factor receptor signalling pathways is both receptor and cell specific and highlights the complexity of HIF-1 regulation by these signalling cascades.

Loss of negative regulators of the PI3K pathway including PTEN, p53 and the tumour-suppressor proteins tuberous sclerosis proteins TSC1 (hamartin), TSC2 (tuberin), and promyelocytic leukemia (PML) leads to hyperactive PI3Ksignalling. PTEN is a phosphatase that dephosphorylates the PI3K products, and thus inhibits PI3K downstream signalling. Loss of PTEN or loss of function of PTEN has been found in a range of cancers including prostate, glioma, and thyroid [53-55]. Loss of PTEN has been associated with enhanced angiogenesis and cancer progression possibly through PI3K-induced HIF- $1 \alpha$ and downstream targets including VEGF. Loss of function or deletion of the tumour suppressor p53 is common in many cancer types including 


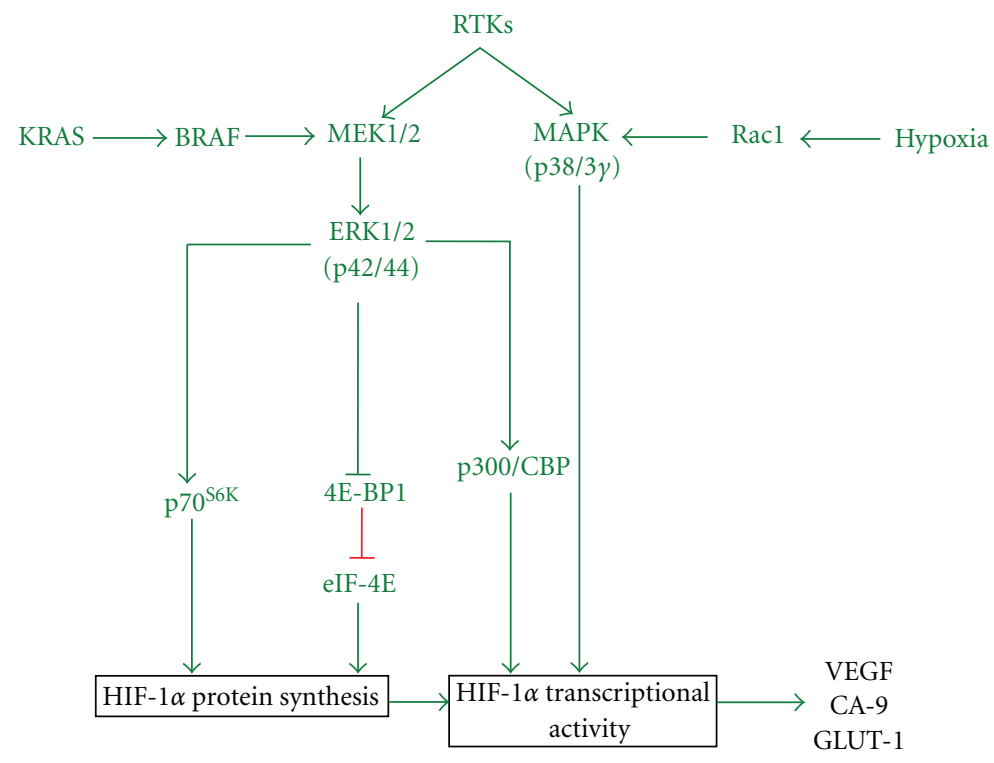

FIGURE 2: Regulation of HIF- $1 \alpha$ by the MAPK pathway. Stimulation of receptor tyrosine kinases (RTKs) by growth factors, or activating mutations in the RAS family of GTPases, such as KRAS, results in activation of a range of MAPK-signalling proteins. Activation of ERK1/2 by MEK1/2 increases HIF-1 signalling (a) by phosphorylating and promoting the interaction of the coactivator p300 with HIF-1 $\alpha$ and (b) by phosphorylating and activating the translational regulatory protein $\mathrm{p} 70^{56 \mathrm{~K}}$ and by phosphorylating and disrupting the repressive interaction of 4E-BP1 with eIF-4E. Activation of these translational machinery proteins leads to increased protein synthesis of HIF- $1 \alpha$. Activation of MAPKs (p38/p38 $)$ ) leads to the direct phosphorylation and activation of HIF-1 $\alpha$. Additionally, under hypoxia, activation of the small GTPase Rac1 leads to a Rac1 dependent increase in HIF- $1 \alpha$ activity via Rac-1 induced activation of p38 MAPK. Genetic mutations in members of the MAPK signalling pathway such as those in BRAF (BRAF ${ }^{\mathrm{V} 600 \mathrm{E}}$ ) lead to hyperactive MEK/ERK signalling and enhanced HIF- $1 \alpha$ activity.

thyroid. p53 negatively regulates the PI3K pathway by inhibiting transcription of the PIK3CA gene that encodes the catalytic subunit of PI3K; p110 $\alpha$ and by activating transcription of PTEN and TSC2 $[56,57]$. Furthermore, p53 can directly interact with HIF- $1 \alpha$ and recruit MDM2, which targets HIF- $1 \alpha$ for ubiquitination and degradation $[28,58]$. mTOR activity is negatively regulated by the heterodimer TSC1 (hamartin) and TSC2 (tuberin). This complex is disrupted and functionally inactivated by AKT leading to enhanced activity of mTOR and upregulation of HIF- $1 \alpha$. This has recently been confirmed in irradiated tumours where HIF- $1 \alpha$ is activated through this pathway [59]. HIF$1 \alpha$ is not only stimulated through PI3K/AKT activation of mTOR via loss of the TSC1/2 complex, but also via mutational changes leading to loss of TCS2 function. Subsequently, HIF- $1 \alpha$ downstream targets including VEGF and Glucose transporter-1(GLUT-1) are increased [60]. Hypoxia inhibits mTOR activity in both a HIF-dependent and independent manner [61]. Under hypoxia, PML negatively regulates mTOR by directly interacting with and preventing activation of mTOR by the small GTPase Rheb1. Loss of PML is observed in a number of sporadic tumours and correlates with increased VEGF and HIF- $1 \alpha$ expression via attenuation of hypoxic mTOR inhibition [62]. Characteristically, patients with mTOR-associated hamartoma and tumours harbouring mutations in TSC2 and PML are highly vascularised, a common phenotype of tumours arising from mutations in VHL, which have hyperactive HIF-1 and VEGF signalling $[60,62]$.
Collectively, these tumour types highlight the importance of HIF-1 in tumour angiogenesis and progression.

Studies in human embryonic kidney cells suggest that mTOR may directly phosphorylate HIF- $1 \alpha$, promoting binding of $\mathrm{p} 300 / \mathrm{CBP}$ and enhancing HIF- $\alpha$ activity. mTOR interacts with the scaffold protein raptor (regulatoryassociated protein of mTOR). Raptor directly interacts with HIF- $1 \alpha$ under conditions favouring stabilisation of HIF- $1 \alpha$, that is, hypoxia, resulting in enhanced activity, which was not due to mTOR induced stabilisation of HIF- $1 \alpha$ protein [63].

The pathophysiology behind increased HIF- $1 \alpha$ in many solid tumours is not only restricted to hypoxia, growth factor signalling cascades, or mutations in components of the oxygen-dependent regulatory mechanism of HIF-1, but also may be mediated by other signalling pathways. A hyperactive $\mathrm{Wnt} / \beta$-catenin signalling cascade may similarly result in increased activity of HIF- 1 due to the interaction of $\beta$-catenin with HIF- $1 \alpha[64,65]$. Collectively, these data highlight that many pathways are involved in the fine tuning of HIF- $1 \alpha$ regulation by oncogenic signalling cascades such as the ERK-MAPK and PI3K/AKT pathways.

\section{The Role of HIF in Cancer}

The majority of solid tumours encounter hypoxic stress as a result of (a) limited oxygen diffusion due to the rapid proliferation of tumour cells which outgrow the existing vascular network and (b) by perfusion deficits mediated by 


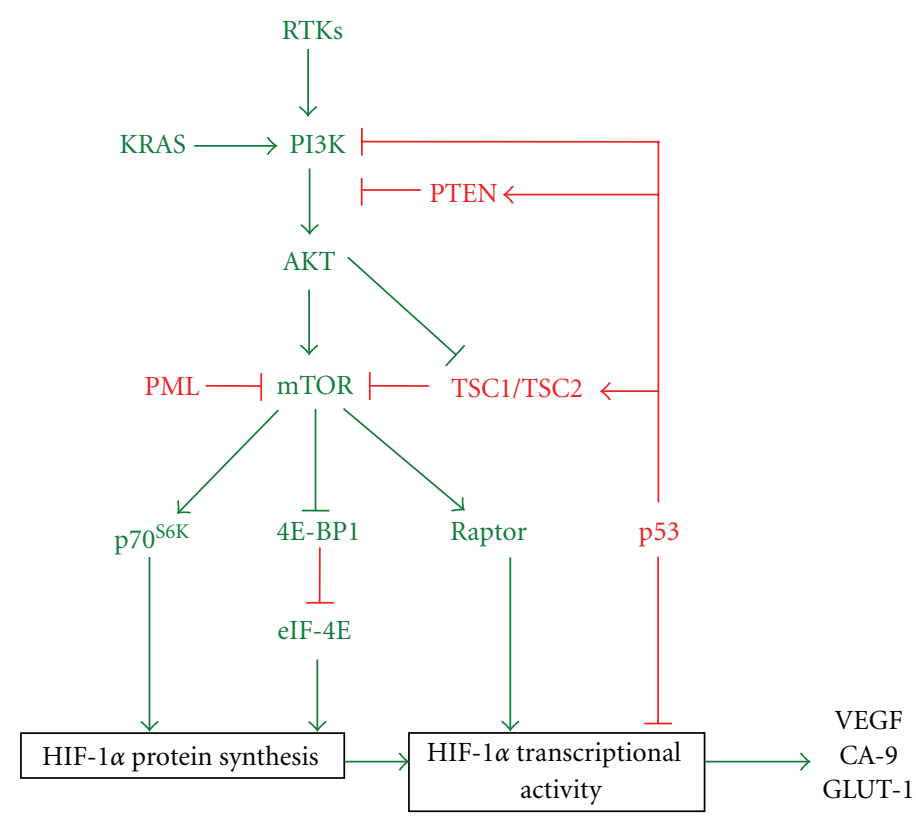

FIgURE 3: Regulation of HIF- $1 \alpha$ by the PI3K pathway. Stimulation of receptor tyrosine kinases (RTKs) by growth factors or activating mutations in the RAS family of GTPases, such as KRAS, results in activation of PI3K. Activated PI3K enables the phosphorylation and activation of AKT. AKT in turn activates mammalian mTOR by disrupting the inhibitory interaction of the TSC1/TSC2 complex with mTOR. mTOR enhances HIF-1 signalling in a multimechanistic way (a) By activating the translational machinery proteins $\mathrm{p} 70^{\mathrm{S} 6 \mathrm{~K}}$ and EIF$4 \mathrm{E}$ leading to enhanced protein synthesis of HIF- $1 \alpha$ and (b) By promoting the interaction of HIF- $1 \alpha$ with the coactivator p300, possibly by direct phosphorylation of HIF- $1 \alpha$ via interaction with the scaffold protein raptor. Additionally, deletion or loss of function of negative regulators of the PI3K pathway leads to enhanced signalling and activation of HIF-1. These include PTEN, p53, PML, and TSC1/2. The phosphatase PTEN dephosphorylates the products of PI3K, inhibiting activation of AKT and subsequent downstream targets. p53 inhibits PI3K signalling at multiple levels; p53 inhibits transcription of the catalytic subunit of PI3K (p110 $\alpha$ ), enhances transcription of PTEN and TSC2, interacts with HIF- $1 \alpha$, and recruits MDM2, targeting HIF- $1 \alpha$ for proteosomal degradation. PML inhibits mTOR under hypoxia and TSC1/2 interacts with and prevents activation of mTOR in the absence of PI3K activation.

abnormal blood vessel structure and function within the tumour $[3,66,67]$. Exposure to low oxygen tensions results in enhanced expression and activity of HIF- $1 \alpha$ leading to increased tumour cell survival and growth under stressful environments. Over one hundred HIF target genes have been identified. HIF promotes tumour cell survival and progression by regulating multiple genes including those involved in angiogenesis (VEGF), anaerobic metabolism (GLUT-1), control of intracellular pH (CA-9), regulation of cell cycle, DNA damage response, proliferation and apoptosis (p21 and p27) and extracellular matrix remodelling and cell migration (lysyl oxidase (LOX), and matrix-metalloprotease1 and -9 (MMP-1 and -9)) [68-71].

Clinically, hypoxia and HIF-1 has been associated with poor prognosis in a range of cancers including uterine, breast, and nonsmall cell lung cancer as well as poor treatment response in cancers such as nasopharyngeal cancer $[1-3,72]$. Additionally, coexpression of HIF- $1 \alpha$ with other pro-oncogenic proteins has been shown to be a significant prognostic predictor of survival. In patients with nonsmall cell lung cancer, coexpression of HIF- $1 \alpha$ with Snail or TWIST1 has been shown to reduce overall survival and recurrence-free survival [73]. Expression of HIF- $2 \alpha$ and coexpression with EGFR and insulin-like growth factor-binding protein-2 (IGFBP-2) has also been linked to reduced survival in patients with higher grade astrocytomas [74].

The HIFs act as the most important sensors of oxygen homeostasis. Constant HIF activation is a common feature in many cancers and is becoming increasingly recognized as a target for therapeutic intervention.

\section{HIF and Chemo/Radiotherapy}

5.1. HIF and Chemoresistance. HIF- $1 \alpha$ has been found to upregulate expression of the ATP-binding cassette (ABC) transporter family of proteins and multidrug resistance related proteins (MRPs) in certain tumours and cancer cell lines. The ABC-transporter proteins can efflux chemotherapeutic agents out of the cell, namely, taxanes or anthracyclines resulting in reduced cytotoxicity and cell death [7577].

The HIF-1 downstream target VEGF has also been implicated in chemoresistance. Blockade of VEGF signalling can promote normalisation of the tumour vasculature resulting in enhanced delivery of chemotherapeutics into the tumour. This has been observed in anaplastic thyroid carcinoma xenografts, where bevacizumab (inhibitory monoclonal antibody against VEGF) lowered tumour interstitial pressure and reduced vascular permeability [78]. Due to enhanced VEGF 
signalling, the tumour vasculature is erratic, dysfunctional, and leaky, resulting in poorly perfused regions and high tumour interstitial pressure. This can hinder diffusion of chemotherapeutics into the tumours [79]. Qayum et al. recently showed that blockade of the EGFR, RAS, and PI3K pathways leads to long-term morphological changes that promote increased perfusion and allow prolonged and enhanced drug delivery to the tumour. These changes may also improve tumour response to radiotherapy [80]. In addition to VEGF being a HIF-1 downstream target, VEGF itself can induce HIF- $1 \alpha$ and promote HIF-1 activity leading to enhanced chemo-resistance.

Other HIF-1 target genes implicated in chemo-resistance include CA-9. CA-9 plays a role in acidification of the tumour microenvironment by catalysing the hydration of $\mathrm{CO}_{2}$ to bicarbonate and protons. Due to this, anticancer drugs that are weakly basic are unable to be taken up effectively into tumour cells as a result of raised intracellular $\mathrm{pH}$ and low extracellular $\mathrm{pH}$, leading to reduced cytotoxicity [81-83]. Additionally, our group has shown that inhibition of HIF- $1 \alpha$ either genetically or pharmacologically can enhance chemosensitivity when used in combination with drugs that lack efficacy in hypoxic cells such as etoposide [84].

Collectively, these data highlight the importance of HIF-1 in chemo-resistance and suggest that combination treatments with HIF inhibitors may be useful in improving therapeutic response to existing drugs and especially those associated with hypoxic chemo-resistance.

5.2. HIF and Radioresistance. Tumour hypoxia is associated with radioresistance due to the lack of oxygen leading to a reduction in the level of radiation-induced free radicals. These free radicals induce single- and double-stranded DNA breaks, leading to cell death by necrosis, apoptosis, or mitotic catastrophe $[85,86]$. Within a tumour, the level of hypoxia has been shown to inversely correlate with radiosensitivity [66]. HIF-1 signalling promotes cell survival following exposure to ionising radiation. Clinically, high HIF-1 activity after radiation is associated with poor prognosis $[2,87]$. HIF1 activity has been found to increase with increasing doses of radiation in tumour xenograft models. No effect of radiation on HIF-1 activity was observed in tumour cells in vitro, suggesting that increased activity is dependent on radiationinduced physical changes within the intact tumour [88]. Conversely, other studies suggest that radiation itself can increase HIF- $1 \alpha$ expression in addition to hypoxia in vitro. HIF-1 reporter activity was found to increase in hypoxic cells following exposure to radiation in range of carcinoma cell lines [89]. As a result of radiation-induced HIF-1 signalling, expression of HIF-1 downstream targets such as VEGF, plasminogen activator inhibitor-1, and CA-9 are increased and promote radio-resistance [90].

Within a tumour, mechanisms thought to contribute to radiation induced increases in HIF-1 include tumour reoxygenation. Radiation increases the level of $\mathrm{O}_{2}$ within a tumour. This is due to cell death of well-oxygenated cells that frees up $\mathrm{O}_{2}$ molecules. Additionally, as there are fewer tumour cells due to cell death, the vasculature is able to expand and grow, leading to enhanced oxygenation. This reoxygenation causes the generation of free radical such as reactive nitrogen species that lead to inhibition of the PHDs, thus reducing proteosomal degradation of HIF- $1 \alpha$ [91].

Hyperactivity of growth factor signalling pathways such as EGFR, RAS, and PI3K/AKT cascades have been linked to radio-resistance and tumour cell survival following radiotherapy [92, 93]. As HIF-1 is a downstream target of PI3K, PI3K-induced HIF-1 activity may contribute to radioresistance. Therefore, it is feasible to assume that tumour cells associated with hyperactive PI3K signaling, and thus, HIF-1 activity may respond better to radiotherapy when used in combination with a PI3K inhibitor. Currently, inhibition at the level of the EGFR in combination with radiotherapy has been used clinically and with success in certain tumour types $[92,94]$. However, as there are many different points of activation within the PI3K signalling pathway, inhibition of PI3K itself may be useful in a whole range of tumours that have hyperactive PI3K signalling which is not dependent on EGFR activation but are dependent on other RTKs. Enhanced radiosensitivity has been observed in colon carcinoma cells treated with the selective PI3K inhibitor PI103 [95] and in glioma xenografts treated with selective small molecule inhibitors of $\mathrm{p} 110 \alpha$ [96]. Thus, these data provide promising evidence that in addition to inhibiting the downstream effects of PI3K that promote cell survival after radiation, indirect targeting of HIF-1 by PI3K inhibitors may significantly contribute to enhanced radiosensitivity.

\section{HIF and Thyroid Hormones}

Thyroid hormones play a key role in growth, metabolism and development. The effects of these hormones are brought about, namely, by the thyroid hormone ligand 3,3,5-triiodothyronine (T3) binding to the nuclear thyroid hormone receptors (TR) $\beta 1, \beta 2$, or $\alpha 1$. The ligand-nuclear receptor complex recruits additional proteins and acts as transcription factors that regulate gene expression by binding to the thyroid hormone response elements (TREs) in the promoter region of target genes. These genomic actions of thyroid hormones usually involve the binding of T3 to monomers, homodimers, or as heterodimers of the TRs with another member of the nuclear hormone receptor family such as the retinoid $\mathrm{X}$ receptor. This results in shedding of corepressors and recruitment of co-activators leading to alterations in transcription of thyroid hormone responsive genes [97, 98]. This contrasts to the rapid, nongenomic actions of thyroid hormones on the activity of ion pumps, cytosolic signalling, mitochondria, and the intracellular protein trafficking from cytosol to nucleus [98].

Thyroid hormones can activate both the PI3K and MAPK-signalling cascades [98-101]. Furthermore, thyroid hormones have been found to directly regulate expression of HIF- $1 \alpha$ via activation of these signal transduction pathways. Microarray analysis of T3 regulated genes in human skin fibroblasts revealed an upregulation of HIF- $1 \alpha$ and HIF1 target genes GLUT1, platelet-type phosphofructokinase (PFK) and monocarboxylate transporter-4 (MCT-4) via 
T3/TR $\beta$ signalling. The specificity of this upregulation by T3 was confirmed in fibroblasts from patients with an inactivating mutation in TR $\beta$. These fibroblasts did not show any such changes in gene expression of HIF- $1 \alpha$ and target genes [99]. The direct upregulation of HIF- $1 \alpha$ by T3 resulted in a T3 indirect increase of GLUT-1, PFK, and MCT-4 expression via enhanced HIF- $1 \alpha$ activity. This direct increase in HIF- $1 \alpha$ activity was due to T3/TR $\beta$ activation of PI3K (Figure 4). Other studies have also shown that a TR $\beta$ mutant $\left(\mathrm{TR} \beta^{\mathrm{PV} / \mathrm{PV}}\right)$ interacts with the PI3K-regulatory subunit $\mathrm{p} 85 \alpha$ leading to enhanced PI3K signalling, promoting thyroid carcinogenesis in a mouse model of spontaneous follicular thyroid cancer (thyroid hormone receptor ${ }^{\mathrm{PV} / \mathrm{PV}}$ mice) [100]. This may lead to enhanced HIF- $1 \alpha$ signalling in vivo.

T3 can also increase HIF- $1 \alpha$ expression through interaction with the $\mathrm{TR} \alpha$ members. In endothelial cells, T3 promotes the interaction of $\mathrm{TR} \alpha 1$ with the PI3K subunit p $85 \alpha$, leading to increased phosphorylation and activation of AKT and endothelial nitric oxide synthase (eNOS). This is thought to contribute to the vasodilatory and cardiovascular protective effects of thyroid hormones [101]. It is likely that this T3-induced PI3K activity in endothelial cells would enhance HIF- $1 \alpha$ activity, which may contribute to the vascular effects of thyroid hormone, as eNOS is a HIF-1 regulated gene [102]. In glioma cells, stimulation of PI3K/AKT by T3 induces shuttling of TR $\alpha$ from the cytoplasm to the nucleus, and promotes transcription of HIF-1 $\alpha$ mRNA (Figure 4). This T3-dependent process was inhibited by treatment with the PI3K inhibitor LY294002 but not by the ERK inhibitor PD098095 [98]. T3-induced expression of HIF1- $\alpha$ and the downstream target VEGF has been verified in a gastric cancer cell line and in vivo mouse model. Accumulation of HIF- $1 \alpha$ was due to T3 dependent activation of the PI3K signalling cascade. Interestingly, in addition to PI3Ksignalling, HIF- $1 \alpha$ overexpression by T3 may be regulated by fumarate accumulation; this accumulation was enhanced by T3-mediated inactivation of fumarate hydratase and reduced in the presence of 2-oxoglutarate [103] (Figure 4). These data link thyroid hormones with metabolic proteins and the HIF1 signalling pathway in carcinogenesis and provide a means for understanding the adaptive mechanisms of tumour cells to metabolic stress.

Interestingly, T4 but not T3 has been found to impact on HIF- $1 \alpha$ expression via interaction with the TR $\beta 1$ in glioblastoma (U-87) cells. This process was dependent on MAPK (ERK1/2) signalling as PD098095 inhibited T4-induced HIF$1 \alpha$ expression [98]. In CV- 1 cells transfected with TR $\beta 1$, T4-activated MAPK signalling led to the interaction of MAPK with TR $\beta 1$ and enhanced phosphorylation and TR $\beta 1$ signalling [104]. Other studies have shown that both T3 and T4 can promote HIF- $1 \alpha$ activity by activating the PI3K pathway. In hepatoma cells, T3 and to a lesser extent T4 increased expression of the HIF-1 target genes EPO and adrenomedullin (ADM), by stimulating the PI3K pathway, thus leading to increased translation of HIF- $1 \alpha$ mRNA. No effects on HIF- $1 \alpha$ protein stability/proteosomal degradation were observed [105].

In addition to the rapid nongenomic induction of HIF$1 \alpha$ by thyroid hormones via activation of growth factor signalling cascades, thyroid hormones have been shown to induce HIF- $1 \alpha$ via the classic genomic actions of TRs. T3 can indirectly increase HIF- $1 \alpha$ mRNA in a range of carcinoma cell lines that express hepatic leukemia factor (HLF). This regulation appears to be independent of a major protein kinase pathway, as the inhibition of members of both the MAPK and PI3K kinase pathways, including mTOR, had no effect on T3-mediated HIF-1 $\alpha$ expression. T3 induction of HIF- $1 \alpha$ was dependent on HLF-mediated gene expression of HIF- $1 \alpha$. This stimulation of HLF was in turn mediated by T3-activation of the TR $\beta$-retinoid X receptor $\alpha$ heterodimer. Increased HIF- $1 \alpha$ was, therefore, solely at the mRNA level and independent of translation [106] (Figure 4).

A close link between thyroid hormones and HIF is further substantiated by data on the activity of deiodinase type 3 (DIO3). Hypoxia induces DIO3, the physiological inactivator of $\mathrm{T} 3$, which catalyses the conversion of $\mathrm{T} 3$ to the metabolically inactive T2 (Figure 4). As this effect was also mimicked by hypoxia mimetics such as cobalt chloride, a direct action of HIF-1 was suspected. HIF- $1 \alpha$ directly interacts with the DIO3 promoter, and thus stimulates DIO3 activity. This, in turn, induces a reduction of T3-dependent activation of metabolic pathways and represents a protective mechanism to reduce metabolic rate in tissues subjected to hypoxia [107].

The effect of T3 and T4 on HIF- $1 \alpha$ is both receptor and cell specific. Furthermore, interaction of these hormones with additional signalling cascades provides insight into the importance of thyroid hormones in driving carcinogenesis by upregulating prosurvival pathways and those involved in the adaptation to the stressful conditions of the tumour microenvironment. An understanding of how these hormones and their receptors differentially regulate HIF- $1 \alpha$ in both normal tissue and cancer may help identify the most appropriate drug treatment to improve therapeutic response.

\section{HIF and Thyroid Cancer}

7.1. Clinical Significance of HIF-1 and Thyroid Carcinomas. Recently we expanded on the potential pathophysiological importance of HIF- $1 \alpha$ in thyroid carcinomas by showing that HIF- $1 \alpha$ protein was variably expressed in primary thyroid carcinomas associated with advancing tumour grade. Tumour samples were representative of papillary (PTC), follicular (FTC), and anaplastic (ATC) thyroid carcinomas [26]. HIF-1 $\alpha$ expression was absent from normal thyroid tissue. Interestingly and consistent with previous findings in a range of cancers, HIF- $1 \alpha$ expression was highest in the most aggressive dedifferentiated anaplastic thyroid carcinomas (ATCs). However, analysis of vessel number and distribution via Von Willebrand factor (vWF) immunostaining were unable to link HIF- $1 \alpha$ expression and vessel distribution. This may have been expected due to the short half-life of HIF- $1 \alpha$ and transient regions of hypoxia that occur as a result of the erratic and dysfunctional vasculature, HIF- $1 \alpha$ expression may not always be detected. Similarly to HIF$1 \alpha$, highest expression of GLUT- 1 was observed in ATCs and cell lines derived from ATCs, with lower expression observed 


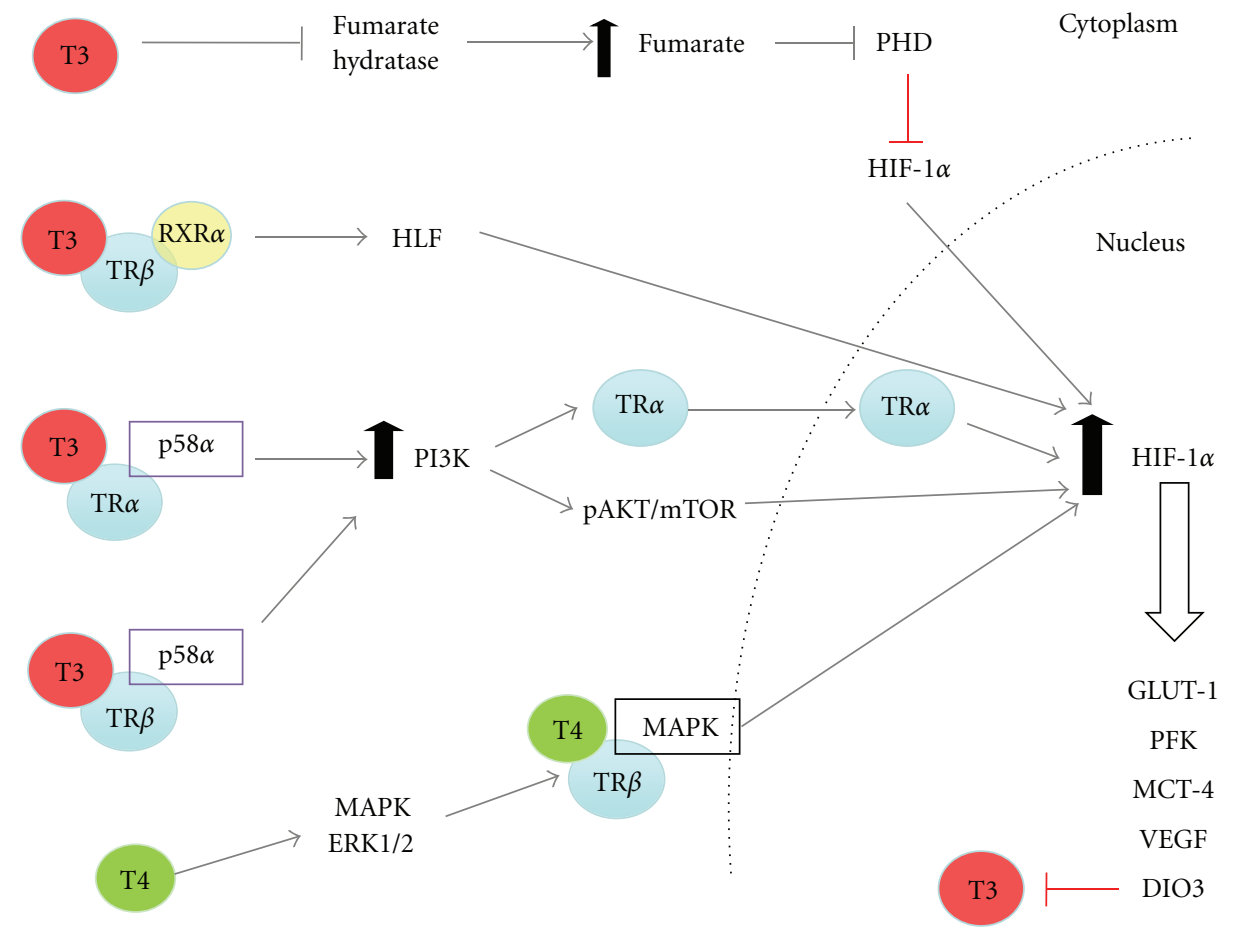

FIgure 4: Thyroid hormone-dependent activation of HIF-1 $\alpha$ : T3 and T4 induce HIF-1 $\alpha$ activity by both genomic and nongenomic mechanisms. Genomically, T3 indirectly increases HIF- $1 \alpha$ mRNA by increasing expression of the transcription factor hepatic leukemia factor (HLF), which initiates transcription of HIF-1 $\alpha$. Non-genomically, T3 stimulates PI3K signalling by promoting the interaction of both TR $\alpha$ and $\beta$ with the PI3K regulatory subunit p85 leading to enhanced PI3K/AKT/mTOR activity and translation of HIF- $1 \alpha$ mRNA. T3induced PI3K promotes nuclear shuttling of TR $\alpha$ leading to increased HIF- $1 \alpha$ expression. T3-induced PI3K signalling by either TR $\alpha$ or $\beta$ is cell specific. T3 inhibits the enzyme fumarate dehydrogenase resulting in the accumulation of fumarate. Fumarate inhibits PHD2 leading to reduced hydroxylation of HIF- $1 \alpha$ and increased protein stabilisation. T4 increases HIF- $1 \alpha$ by stimulating MAPK signalling, leading to enhanced T4/TR $\beta$ activity and expression of HIF- $1 \alpha$. Activated HIF- $1 \alpha$ by T3/T4 results in the upregulation of target genes, known to promote tumour cell survival and progression. These include GLUT-1, PFK, MCT-4, and VEGF. Additionally, HIF-1 upregulates DIO3, which inhibits $\mathrm{T} 3$ by catalysing the conversion of $\mathrm{T} 3$ to the metabolically inactive $\mathrm{T} 2$.

in cell lines derived from differentiated tumours. These increases, however, were only slight and were not significant. In contrast to GLUT-1, the HIF-1 downstream target CA9 closely mimicked the pattern of HIF- $1 \alpha$ expression with significantly higher levels observed in the ATCs. This suggests that CA-9 as in other cancer types may serve as a potential new biomarker of aggressive disease in thyroid carcinomas. This observation has been supported by previous work by Jubb et al. who reported high expression levels of HIF$1 \alpha$ in all thyroid carcinoma types particularly in FTCs $[29,108]$. Additionally, data from a preliminary study in our group looking at HIF- $1 \alpha$ and CA-9 in PTC, FTC, and ATC xenografts showed a similar correlation to that seen in clinical samples; both HIF- $1 \alpha$ and CA- 9 expression was highest in the tumours derived from ATC (8505c) cells. Furthermore, tumours with high expression of HIF- $1 \alpha$ and CA-9 had a greater number of spontaneous metastatic colonies to the lungs (Figure 5). Collectively, these data from primary tumour tissue and xenografts suggest that HIF- $1 \alpha$ expression is involved in the adaption of thyroid carcinomas to hypoxia and supports a pathophysiological role for thyroid tumour progression, aggressiveness and metastasis.
7.2. Hypoxia and HIF-1 in Thyroid Carcinomas. A number of functional studies in thyroid carcinoma cell lines and in immortalized cells derived from normal thyroid tissue substantiate the importance of hypoxia-induced HIF-1 in promoting the expression of proteins that drive thyroid tumour progression. HIF- $1 \alpha$ was predominantly localized in the cytoplasm under normoxia but stabilised and translocated to the nucleus in hypoxia [29]. Enhanced nuclear localisation of HIF- $1 \alpha$ has also been observed in the BcPAP cell line harbouring the $\mathrm{BRAF}^{\mathrm{V} 600 \mathrm{E}}$ mutation versus a cell line with WT BRAF under normoxia [30]. The functional response of both normal and carcinoma thyroid cell lines to hypoxia was further confirmed as graded hypoxia-induced a marked increase in expression of HIF- $1 \alpha$ and downstream targets CA-9, VEGF and GLUT1. Hypoxia induced HIF$1 \alpha$ was further supported by HIF- $1 \alpha$ reporter activation in cells exposed to lowering oxygen tensions. Furthermore, the degree of hypoxia induced HIF- $1 \alpha$ activity related to the level of thyroid carcinoma aggressiveness; lowest activity was observed in PTC cell lines, with highest seen in ATC cells. We have also confirmed that hypoxia induces expression of HIF- $2 \alpha$ in follicular and anaplastic thyroid carcinoma cell lines (unpublished, data not shown). This is of interest, 

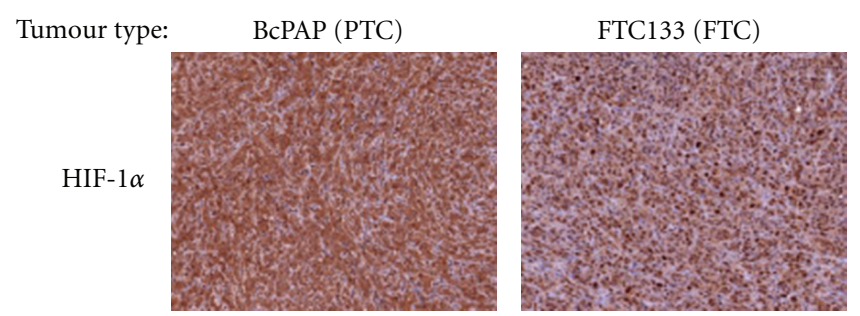

(a)
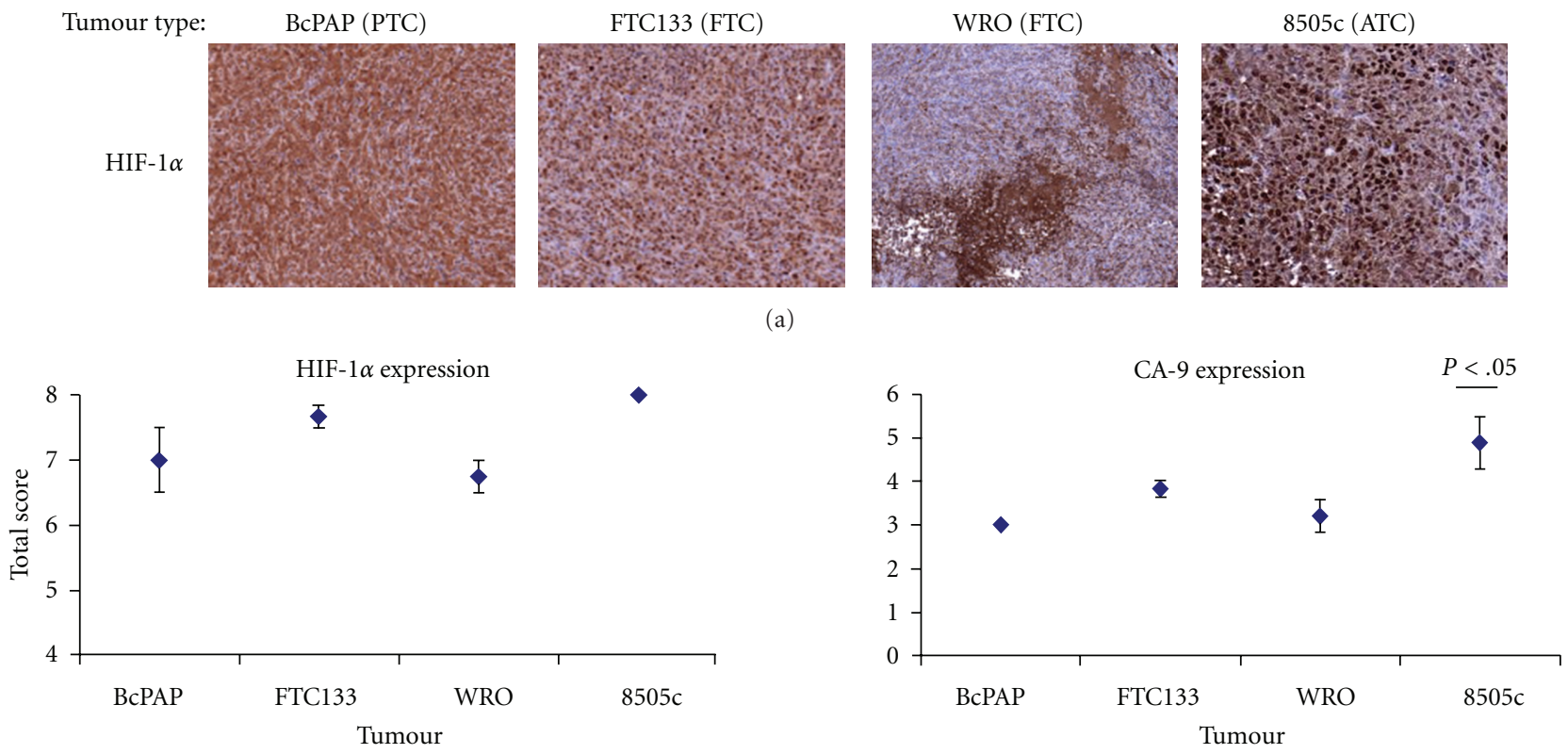

(b)

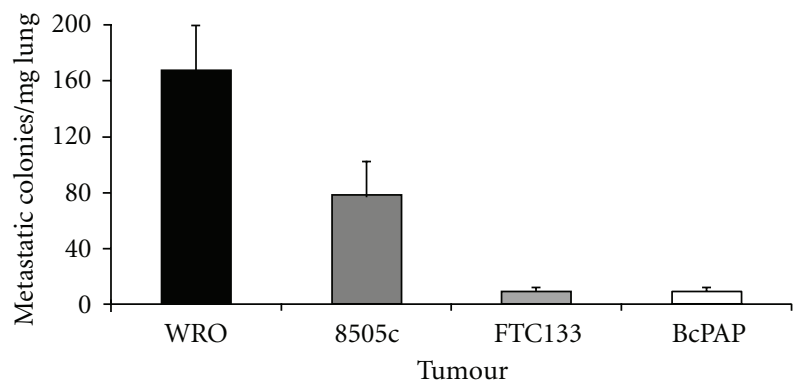

(c)

FIGURE 5: HIF- $1 \alpha$ and CA-9 protein expression and metastatic potential are highest in tumours derived from follicular (WRO) and anaplastic (8505c) cell lines. Briefly, BcPAP, FTC133, WRO and 8505c cells were implanted sub-cutaneously in female CBA nu/nu nude mice. Once tumours reached $450 \mathrm{~mm}^{3}$, tumour tissue and fresh lung tissue were excised. Tumour sections were immuno-stained for HIF-1 $\alpha$. Freshly excised lung tissue was enzymatically digested, plated out in serial dilution and left until visible colonies formed. Colonies were stained, counted and number of metastatic colonies per mg lung tissue calculated. (For full methods, see supplementary materials and methods which is located at doi: 10.406/2011/762905). (a) Immunohistochemical staining for HIF-1 $\alpha$ (brown staining). One example of HIF- $1 \alpha$ staining for each tumour type: BcPAP, FTC133, WRO and 8505c are displayed. (b) Semiquantitative analysis of HIF- $1 \alpha$ and CA-9 immunostaining quantified according to Allred et al. [109]. The total score is representative of intensity and proportion of staining. HIF-1 $\alpha$ was highly expressed in all tumour types with highest expression observed in the 8505c tumours. CA-9 was also expressed in all tumour types with significantly higher expression observed in $8505 \mathrm{c}$ tumours $(P<.05$, one way Anova, with Tukey post test). (c) The number of spontaneous metastatic colonies cultured from enzymatically digested lung tissue that was excised from mice bearing WRO, 8505c, FTC133, and BcPAP tumours. The highest number of colonies counted were from the lungs of mice bearing FTC (WRO) and ATC (8505c) tumours. Data are representative of the mean \pm S.D. of 5 independent mice for each tumour type.

as the HIFs are known to regulate genes differentially in many different cancer types. Therefore, expression in thyroid carcinoma may account for some of the phenotypic differences seen between the different classifications of thyroid carcinoma.

7.3. Growth-Factor Signalling Pathways and HIF-1 in Thyroid Carcinomas. We found that in addition to hypoxia, growth factor-signalling pathways also induced HIF- $1 \alpha$ expression and activity in thyroid carcinoma cell lines. Induction of HIF- $1 \alpha$ by pathways such as the MAPK and PI3K signalling cascades have been described in a range of cancer types (see oxygen-independent regulation of HIF) [110]. The majority of thyroid carcinomas have mutations in growth factor signalling pathways. Mutations in the BRAF gene (BRAF ${ }^{\mathrm{V} 600 \mathrm{E}}$ ) leading to hyperactive MAPK/ERK signalling have been found in approximately 50\% of all PTCs [38]. Recently, this mutation has been reported to increase the transcription and expression of HIF- $1 \alpha$ protein [30].

KRAS has been found to differentially regulate HIFs in certain cancer types [44]. Activating mutations in the RAS family of GTPases, such as those found in KRAS, 


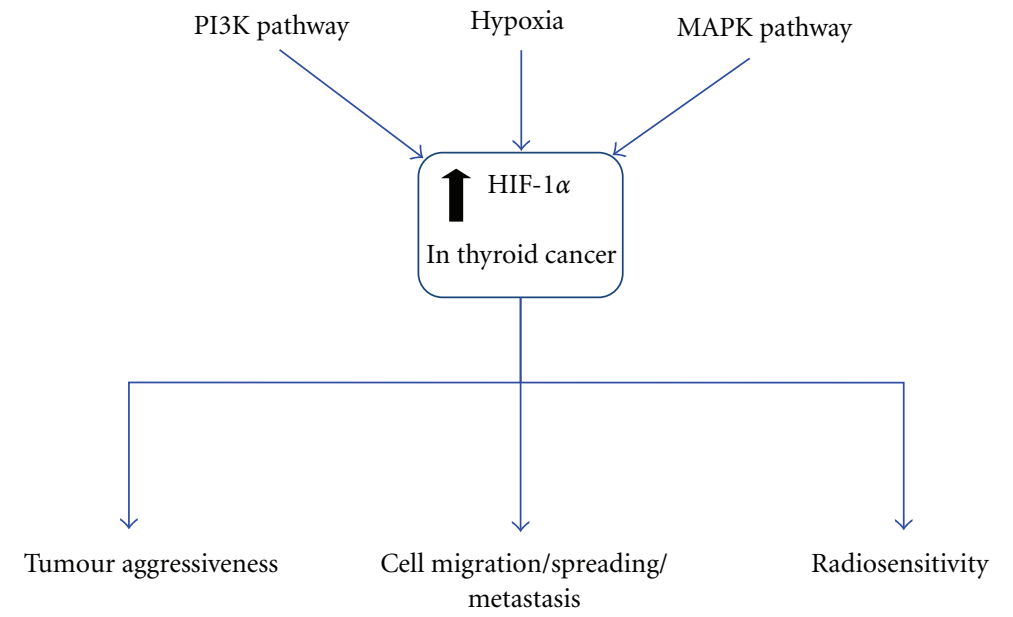

FIGURE 6: Mechanisms of induction of HIF- $1 \alpha$ and downstream effects of HIF-1 signalling in thyroid carcinomas. HIF-1 activity is induced not only by hypoxia but by the oncogenic MAPK and PI3K signalling pathways. Clinically, expression of HIF- $1 \alpha$ and the downstream target CA-9 have been linked with thyroid carcinoma aggressiveness. It is well known that HIF-1 plays an important role in tumour development, metastasis, and aggressiveness and is ultimately correlated with poor prognosis. Enhanced HIF-1 signalling by hypoxia and the PI3K/MAPK pathways may play an important role in the metastatic characteristics of thyroid carcinoma. HIF-1-induced MET upregulation has been implicated in promoting invasiveness of PTC. HIF-1 signalling may also play a prominent role in the therapeutic response to radiotherapy in thyroid carcinoma.

have been found in approximately $20 \%$ of PTCs and more specifically the follicular variant of PTC suggesting the mutation promotes aggressive phenotype [43]. As KRAS activates both BRAF and PI3K, it would be interesting to determine the role of KRAS in the context of HIF activity and aggressive phenotype in thyroid tumours and thyroid carcinoma cell lines harbouring this mutation.

Studies have shown that highest levels of HIF- $1 \alpha$ expression and activity were detected in thyroid tumours and tumour cell lines harbouring classic mutations in BRAF and PI3K $[29,30]$. Thus, the current data supports the importance of oncogenic activation of HIF-1 in thyroid cancer and aggressive disease [46]. Direct intervention with pharmacological inhibitors of these pathways (LY294002 for PI3K, PD098059 for ERK/MAPK, and sorafenib for inhibition of RAF-1 kinase/BRAF) or by genetic modulation; (reexpression of PTEN in PTEN null cell lines and silencing of mutant BRAF), further support the close dependency of HIF expression on oncogenic signalling. Interestingly, inhibition of HIF- $1 \alpha$ reporter activity was much more substantial as a consequence of LY294002 treatment under varying $\mathrm{O}_{2}$ tensions compared to PD98059 even in cell lines with no known mutation in the PI3K pathway. These findings are particularly interesting with regard to the frequent mutations in the PI3K/AKT pathway found in up to $15 \%$ of PTC and approximately $50 \%$ of FTC $[111,112]$, where PI3Kpathway mutations have been directly linked to tumour aggressiveness [41]. HIF- $1 \alpha$ silencing reduced expression of HIF-1 target genes like CA-9. Thus, blocking HIF- $1 \alpha$, either directly or through inhibition of the PI3K pathway in thyroid carcinomas, may decrease aggressiveness and thus open new therapeutic options [46].
7.4. HIF and VEGF in Thyroid Carcinomas. VEGF serum levels are elevated in thyroid carcinomas, and this is associated with poor tumour prognosis [113-116]. As one of the most important downstream targets of HIF-1 signalling, VEGF has not yet been linked clinically to HIF activity in thyroid carcinomas. A number of studies using antagonists of VEGF signalling for the treatment of advanced thyroid carcinomas have been reported with some positive results ([117-120]. However, no data are currently available on the effect on VEGF levels by direct targeting of HIF-1. We have reported that VEGF levels are significantly increased by anoxia in thyroid carcinoma cells lines derived from PTC, FTC and ATC. Additionally, highest basal levels of VEGF were observed in the PTEN-null FTC133 follicular thyroid carcinoma cell lines [29]. This suggests that the two different signalling pathways may act synergistically to increase VEGF. The anoxia-induced increases in VEGF expression did not, however, match the pattern of HIF- $1 \alpha$ reporter activity in varying thyroid carcinoma cell lines. This suggests that the level of dependency on either the HIF1 or growth factor-signalling pathways to induce VEGF is specific to the type of thyroid carcinoma cell line. Current unpublished data from our group supports a prominent role and an important link between the PI3K and HIF1 pathways in the regulation of VEGF. Furthermore, we have found that inhibition of these pathways has profound effects on VEGF expression in thyroid carcinoma cell lines. These data provide a new angle for therapeutic approaches of thyroid carcinoma as drugs which target HIF-1 signalling may be more efficacious in downregulating VEGF dependent signalling in these tumours. 
7.5. HIF-1, Migration, and Metastasis in Thyroid Carcinomas. Hypoxia and HIF-1 have been associated with increased migration and metastasis in a range of cancer types [121123]. We have found that hypoxia significantly increased migration in cell lines derived from PTCs and FTCs. Additionally, we have exciting data that supports a prominent role of PI3K and HIF-1 signalling in metastatic characteristics of FTC and ATC (unpublished).

Studies have shown a link between hypoxia/HIF- $1 \alpha$ and MNNG HOS transforming gene (MET) upregulation in clinical PTCs. MET is a receptor tyrosine kinase that is stimulated by hepatocyte growth factor (HGF). Stimulation results in the activation of signal transduction pathways such as PI3K, which promote migration, invasion, and the release of cytokines and proangiogenic factors such as VEGF [124, 125]. Additionally, MET activation promotes migration and invasion by stimulating the translocation of $\beta$-catenin from the cytoplasm to the nucleus [126]. MET is highly expressed in PTC, and this high expression of MET is thought to be due to HIF-1 induced upregulation in hypoxic regions of PTCs. Furthermore, higher expression of MET and HIF- $1 \alpha$ mRNA was found at the periphery of tumours (in cells located at the invading front) compared to the centre in $44 \%$ of PTCs [127]. These were characterised to have a more aggressive phenotype. Collectively, these data suggest that HIF-1 may play a role in promoting migration and aggressiveness in PTC.

Taken together, these data highlight the importance of hypoxia, HIF- $1 \alpha$ and PI3K signalling as a means of promoting metastatic characteristics and driving aggressive disease in thyroid carcinomas (Figure 6).

\section{HIF-1 and Sensitivity to Radiotherapy in Thyroid Carcinomas}

There is increasing evidence for the role of HIF in tumour cell sensitivity to chemo- and importantly radiotherapy [77, 84, 128]. As discussed above, HIF-1 stabilisation and action depend on growth factor-signalling pathways. Thus, many inhibitors of growth factor signalling affect HIF-1 expression and downstream targets. Anticancer drugs that target these pathways possess antiangiogenic properties and include the BCR-ABL kinase inhibitor imatinib (Gleevec), EGFR inhibitors such as gefitinib, erlotinib, and cetuximab, the HER2 ${ }^{\text {neu }}$ inhibitor trastuzumab, and the mTOR inhibitors rapamycin, temsirolimus, and everolimus. Collectively, these drugs all target the translation of HIF- $1 \alpha$ mRNA into protein [129]. Modulation of HIF-1 $\alpha$ expression can increase tumour sensitivity, in particular to radiotherapy in certain cancer types (see HIF and radio-resistance). Thus, HIF-1 signalling may be a particularly important feature for the sensitivity of dedifferentiated thyroid carcinomas to external beam radiation. This has yet to be investigated.

It has been shown that tumour cell apoptosis is increased when targeted radioiodide therapy is combined with angiogenic inhibitors, which represents an indirect approach to circumvent HIF-1—dependent radio-resistance [130]. Data discussed here support the view that targeting of HIF-1 may serve as a more direct approach to overcome HIF dependent adaptation of thyroid carcinoma cells to radiotherapy. Preliminary data from our group in FTC and ATC cell lines supports this notion of HIF-1 signalling playing a role in radio-resistance (unpublished). As described earlier, PI3K has been found to play an important role in HIF- $1 \alpha$ induced expression and activity in thyroid carcinoma cell lines under both normoxia and anoxia. Therefore, targeting HIF-1 either directly or by use of a PI3K-inhibitor may improve the therapeutic response of thyroid carcinoma to radiotherapy.

Currently, there is no data available on whether inhibition of HIF-1 may reflect on the sensitivity of more differentiated tumours to standard radiotherapy. It is conceivable that in patients receiving external beam radiation treatment and particularly in patients with a poor response but positive uptake of radioiodine, any augmentation of the sensitivity towards irradiation-induced apoptosis would be favourable (Figure 6). Studies in our group on animal models of metastatic follicular and anaplastic thyroid carcinomas will hopefully allow us to answers some of these questions in the future.

\section{Conclusions}

HIF-1 is potently induced by both hypoxia and oncogenic signalling pathways in thyroid carcinoma, and its expression and activity have been correlated with aggressiveness. Current literature suggests that PI3K and MAPK pathways promote aggressive and metastatic disease in part via the upregulation of HIF-1 activity. With the known effects of hypoxia, PI3K/MAPK pathways, and HIF-1 on desensitisation to radiotherapy, HIF-1 may be a new and important therapeutic target in reducing local tumour growth, metastatic burden, and radio-resistance in thyroid carcinoma.

\section{Financial Support}

Cancer Research UK (K. J. Williams C7820/A8696; supporting N. Burrows) EU FP7 Metoxia Grant agreement no. 222741 (K. J. Williams supporting M. Babur), and Wellcome Trust project grant, UK 082794 (G. Brabant, supporting J. Resch).

\section{Acknowledgment}

K. J. Williams and G. Brabant contributed equally to the work.

\section{References}

[1] P. Birner, M. Schindl, A. Obermair, G. Breitenecker, and G. Oberhuber, "Expression of hypoxia-inducible factor lalpha in epithelial ovarian tumors: its impact on prognosis and on response to chemotherapy," Clinical Cancer Research, vol. 7, no. 6, pp. 1661-1668, 2001.

[2] D. M. Aebersold, P. Burri, K. T. Beer et al., "Expression of hypoxia-inducible factor-1alpha: a novel predictive and prognostic parameter in the radiotherapy of oropharyngeal cancer," Cancer Research, vol. 61, no. 7, pp. 2911-2916, 2001. 
[3] M. Höckel and P. Vaupel, "Tumor hypoxia: definitions and current clinical, biologic, and molecular aspects," Journal of the National Cancer Institute, vol. 93, no. 4, pp. 266-276, 2001.

[4] M. C. Brahimi-Horn and J. Pouysségur, "Harnessing the hypoxia-inducible factor in cancer and ischemic disease," Biochemical Pharmacology, vol. 73, no. 3, pp. 450-457, 2007.

[5] K. Hirota and G. L. Semenza, "Regulation of hypoxiainducible factor 1 by prolyl and asparaginyl hydroxylases," Biochemical and Biophysical Research Communications, vol. 338, no. 1, pp. 610-616, 2005.

[6] J. Pouysségur and F. Mechta-Grigoriou, "Redox regulation of the hypoxia-inducible factor," Biological Chemistry, vol. 387, no. 10-11, pp. 1337-1346, 2006.

[7] S. A. Patel and M. C. Simon, "Biology of hypoxia-inducible factor- $2 \alpha$ in development and disease," Cell Death \& Differentiation, vol. 15, no. 4, pp. 628-634, 2008.

[8] A. Loboda, A. Jozkowicz, and J. Dulak, "HIF-1 and HIF-2 transcription factors-similar but not identical," Molecules \& Cells, vol. 29, no. 5, pp. 435-442, 2010.

[9] O. Aprelikova, M. Wood, S. Tackett, G. V. R. Chandramouli, and J. C. Barrett, "Role of ETS transcription factors in the hypoxia-inducible factor-2 target gene selection," Cancer Research, vol. 66, no. 11, pp. 5641-5647, 2006.

[10] C. J. Hu, LI. Y. Wang, L. A. Chodosh, B. Keith, and M. C. Simon, "Differential roles of hypoxia-inducible factor 1alpha (HIF-1alpha) and HIF-2alpha in hypoxic gene regulation," Molecular and Cellular Biology, vol. 23, no. 24, pp. 9361-9374, 2003.

[11] C. Warnecke, Z. Zaborowska, J. Kurreck et al., "Differentiating the functional role of hypoxia-inducible factor (HIF)-1alpha and HIF-2alpha (EPAS-1) by the use of RNA interference: erythropoietin is a HIF-2alpha target gene in Hep3B and Kelly cells," The FASEB Journal, vol. 18, no. 12, pp. 1462-1464, 2004.

[12] L. Holmquist-Mengelbier, E. Fredlund, T. Löfstedt et al., "Recruitment of HIF-1alpha and HIF-2alpha to common target genes is differentially regulated in neuroblastoma: HIF2alpha promotes an aggressive phenotype," Cancer Cell, vol. 10, no. 5, pp. 413-423, 2006.

[13] T. Tanaka, M. Wiesener, W. Bernhardt, K. U. Eckardt, and C. Warnecke, "The human HIF (hypoxia-inducible factor)3alpha gene is a HIF-1 target gene and may modulate hypoxic gene induction," Biochemical Journal, vol. 424, no. 1, pp. 143 $151,2009$.

[14] P. H. Maxwell, M. S. Wlesener, G. W. Chang et al., "The tumour suppressor protein VHL targets hypoxia-inducible factors for oxygen-dependent proteolysis," Nature, vol. 399, no. 6733, pp. 271-275, 1999.

[15] J. R. Gnarra, J. M. Ward, F. D. Porter et al., "Defective placental vasculogenesis causes embryonic lethality in VHLdeficient mice," Proceedings of the National Academy of Sciences of the United States of America, vol. 94, no. 17, pp. 9102-9107, 1997.

[16] S. C. Clifford, M. E. Cockman, A. C. Smallwood et al., "Contrasting effects on HIF-1alpha regulation by disease-causing pVHL mutations correlate with patterns of tumourigenesis invon Hippel-Lindau disease," Human Molecular Genetics, vol. 10, no. 10, pp. 1029-1038, 2001.

[17] P. P. Kapitsinou and V. H. Haase, "The VHL tumor suppressor and HIF: insights from genetic studies in mice," Cell Death \& Differentiation, vol. 15, no. 4, pp. 650-659, 2008.
[18] C. J. Schofield and P. J. Ratcliffe, "Signalling hypoxia by HIF hydroxylases," Biochemical and Biophysical Research Communications, vol. 338, no. 1, pp. 617-626, 2005.

[19] W. M. Linehan, R. Srinivasan, and L. S. Schmidt, "The genetic basis of kidney cancer: a metabolic disease," Nature Reviews Urology, vol. 7, no. 5, pp. 277-285, 2010.

[20] P. J. Pollard, N. C. Wortham, and I. P. Tomlinson, “The TCA cycle and tumorigenesis: the examples of fumarate hydratase and succinate dehydrogenase," Annals of Medicine, vol. 35, no. 8, pp. 632-639, 2003.

[21] M. A. Selak, S. M. Armour, E. D. MacKenzie et al., "Succinate links TCA cycle dysfunction to oncogenesis by inhibiting HIF-alpha; prolyl hydroxylase," Cancer Cell, vol. 7, no. 1, pp. 77-85, 2005.

[22] E. Gottlieb and I. P. Tomlinson, "Mitochondrial tumour suppressors: a genetic and biochemical update," Nature Reviews Cancer, vol. 5, no. 11, pp. 857-866, 2005.

[23] B. Pasini and C. A. Stratakis, "SDH mutations in tumorigenesis and inherited endocrine tumours: lesson from the phaeochromocytoma-paraganglioma syndromes," Journal of Internal Medicine, vol. 266, no. 1, pp. 19-42, 2009.

[24] J. A. Fagin and N. Mitsiades, "Molecular pathology of thyroid cancer: diagnostic and clinical implications," Best Practice \& Research Clinical Endocrinology \& Metabolism, vol. 22, no. 6, pp. 955-969, 2008.

[25] D. Roymans and H. Slegers, "Phosphatidylinositol 3-kinases in tumor progression," European Journal of Biochemistry, vol. 268, no. 3, pp. 487-498, 2001.

[26] M. Hanada, J. Feng, and B. A. Hemmings, "Structure, regulation and function of $\mathrm{PKB} / \mathrm{AKT}-\mathrm{A}$ major therapeutic target," Biochimica et Biophysica Acta, vol. 1697, no. 1-2, pp. 3-16, 2004.

[27] E. Berra, J. Milanini, D. E. Richard et al., "Signaling angiogenesis via p42/p44 MAP kinase and hypoxia," Biochemical Pharmacology, vol. 60, no. 8, pp. 1171-1178, 2000.

[28] G. L. Semenza, "HIF-1 and tumor progression: pathophysiology and therapeutics," Trends in Molecular Medicine, vol. 8, no. 4, supplement, pp. S62-S67, 2002.

[29] N. Burrows, J. Resch, R. L. Cowen et al., "Expression of hypoxia-inducible factor lalpha in thyroid carcinomas," Endocrine-Related Cancer, vol. 17, no. 1, pp. 61-72, 2010.

[30] M. Zerilli, G. Zito, A. Martorana et al., "BRAF mutation influences hypoxia-inducible factor-1alpha expression levels in papillary thyroid cancer," Modern Pathology, vol. 28, no. 3, pp. 1052-1060, 2010.

[31] N. Sang, D. P. Stiehl, J. Bohensky, I. Leshchinsky, V. Srinivas, and J. Caro, "MAPK signaling up-regulates the activity of hypoxia-inducible factors by its effects on p300," The Journal of Biological Chemistry, vol. 278, no. 16, pp. 14013-14019, 2003.

[32] D. E. Richard, E. Berra, E. Gothié, D. Roux, and J. Pouysségur, "p42/p44 mitogen-activated protein kinases phosphorylate hypoxia-reducible factor (HIF-1alpha) and enhance the transcriptional activity of HIF-1," The Journal of Biological Chemistry, vol. 274, no. 46, pp. 32631-32637, 1999.

[33] K. Hirota and G. L. Semenza, "Rac1 Activity is Required for the Activation of Hypoxia-inducible Factor 1," The Journal of Biological Chemistry, vol. 276, no. 24, pp. 21166-21172, 2001.

[34] G. L. Semenza, "Signal transduction to hypoxia-inducible factor 1," Biochemical Pharmacology, vol. 64, no. 5-6, pp. 993998, 2002. 
[35] A. Görlach, U. Berchner-Pfannschmidt, C. Wotzlaw et al., "Reactive oxygen species modulate HIF-I mediated PAI-I expression: involvement of the GTPase RacI," Thrombosis \& Haemostasis, vol. 89, no. 5, pp. 926-935, 2003.

[36] I. Mylonis, G. Chachami, M. Samiotaki et al., "Identification of MAPK phosphorylation sites and their role in the localization and activity of hypoxia-inducible factor-1alpha," The Journal of Biological Chemistry, vol. 281, no. 44, pp. 33095-33106, 2006.

[37] R. Fukuda, K. Hirota, F. Fan, Y. D. Jung, L. M. Ellis, and G. L. Semenza, "Insulin-like growth factor 1 induces hypoxiainducible factor 1-mediated vascular endothelial growth factor expression, which is dependent on MAP kinase and phosphatidylinositol 3-kinase signaling in colon cancer cells," The Journal of Biological Chemistry, vol. 277, no. 41, pp. 38205-38211, 2002.

[38] K. T. Tang and C. H. Lee, "BRAF mutation in papillary thyroid carcinoma: pathogenic role and clinical implications," Journal of the Chinese Medical Association, vol. 73, no. 3, pp. 113-128, 2010.

[39] R. Ciampi and Y. E. Nikiforov, "Minireview: RET/PTC rearrangements and braf mutations in thyroid tumorigenesis," Endocrinology, vol. 148, no. 3, pp. 936-941, 2007.

[40] Y. Li, M. Nakamura, and K. Kakudo, "Targeting of the BRAF gene in papillary thyroid carcinoma (review)," Oncology Reports, vol. 22, no. 4, pp. 671-681, 2009.

[41] J. E. Paes and M. D. Ringel, "Dysregulation of the phosphatidylinositol 3-kinase pathway in thyroid neoplasia," Endocrinology and Metabolism Clinics of North America, vol. 37, no. 2, pp. 375-387, 2008.

[42] A. T. Franco, R. Malaguarnera, S. Refetoff et al., "Thyrotrophin receptor signaling dependence of Braf-induced thyroid tumor initiation in mice," Proceedings of the National Academy of Sciences of the United States of America, vol. 108, no. 4, pp. 1615-1620, 2011.

[43] A. Greco, M. G. Borrello, C. Miranda, D. Degl'Innocenti, and M. A. Pieroiti, "Molecular pathology of differentiated thyroid cancer," Quarterly Journal of Nuclear Medicine and Molecular Imaging, vol. 53, no. 5, pp. 440-454, 2009.

[44] H. Kikuchi, M. S. Pino, Z. Min, S. Shirasawa, and D. C. Chung, "Oncogenic KRAS and BRAF differentially regulate hypoxia-inducible factor-1alpha and -2alpha in colon cancer," Cancer Research, vol. 69, no. 21, pp. 8499-8506, 2009.

[45] P. Papageorgis, K. Cheng, S. Ozturk et al., "Smad4 inactivation promotes malignancy and drug resistance of colon cancer," Cancer Research, vol. 71, no. 3, pp. 998-1008, 2011.

[46] J. I. Bärdos and M. Ashcroft, "Hypoxia-inducible factor-1 and oncogenic signalling," BioEssays, vol. 26, no. 3, pp. 262269, 2004.

[47] M. Saji and M. D. Ringel, “The PI3K-Akt-mTOR pathway in initiation and progression of thyroid tumors," Molecular \& Cellular Endocrinology, vol. 321, no. 1, pp. 20-28, 2010.

[48] A. C. Gingras, B. Raught, and N. Sonenberg, "Regulation of translation initiation by FRAP/mTOR," Genes \& Development, vol. 15, no. 7, pp. 807-826, 2001.

[49] R. T. Peterson, B. N. Desai, J. S. Hardwick, and S. L. Schreiber, "Protein phosphatase $2 \mathrm{~A}$ interacts with the 70$\mathrm{kDa} S 6$ kinase and is activated by inhibition of FKBP12rapamycin-associated protein," Proceedings of the National Academy of Sciences of the United States of America, vol. 96, no. 8, pp. 4438-4442, 1999.
[50] E. Laughner, P. Taghavi, K. Chiles, P. C. Mahon, and G. L. Semenza, "HER2 (neu) signaling increases the rate of hypoxia-inducible factor lalpha (HIF-1alpha) synthesis: novel mechanism for HIF-1-mediated vascular endothelial growth factor expression," Molecular \& Cellular Biology, vol. 21, no. 12, pp. 3995-4004, 2001.

[51] H. Zhong, K. Chiles, D. Feldser et al., "Modulation of hypoxia-inducible factor lalpha expression by the epidermal growth factor/phosphatidylinositol 3kinase/PTEN/AKT/FRAP pathway in human prostate cancer cells: implications for tumor angiogenesis and therapeutics," Cancer Research, vol. 60, no. 6, pp. 1541-1545, 2000.

[52] A. A. Kazi and R. D. Koos, "Estrogen-induced activation of hypoxia-inducible factor-1alpha, vascular endothelial growth factor expression, and edema in the uterus are mediated by the phosphatidylinositol 3-kinase/Akt pathway," Endocrinology, vol. 148, no. 5, pp. 2363-2374, 2007.

[53] N. D. Deocampo, H. Huang, and D. J. Tindall, "The role of PTEN in the progression and survival of prostate cancer," Minerva Endocrinologica, vol. 28, no. 2, pp. 145-153, 2003.

[54] W. Zundel, C. Schindler, D. Haas-Kogan et al., "Loss of PTEN facilitates HIF-1-mediated gene expression," Genes and Development, vol. 14, no. 4, pp. 391-396, 2000.

[55] C. Eng, "Role of PTEN, a lipid phosphatase upstream effector of protein kinase B, in epithelial thyroid carcinogenesis," Annals of the New York Academy of Sciences, vol. 968, pp. 213221, 2002.

[56] A. Astanehe, D. Arenillas, W. W. Wasserman et al., "Mechanisms underlying p53 regulation of PIK3CA transcription in ovarian surface epithelium and in ovarian cancer," Journal of Cell Science, vol. 121, no. 5, pp. 664-674, 2008.

[57] M. Cully, H. You, A. J. Levine, and T. W. Mak, "Beyond PTEN mutations: the PI3K pathway as an integrator of multiple inputs during tumorigenesis," Nature Reviews Cancer, vol. 6, no. 3, pp. 184-192, 2006.

[58] R. Ravi, B. Mookerjee, Z. M. Bhujwalla et al., "Regulation of tumor angiogenesis by $\mathrm{p} 53$-induced degradation of hypoxiainducible factor lalpha," Genes \& Development, vol. 14, no. 1, pp. 34-44, 2000.

[59] H. Harada, S. Itasaka, S. Kizaka-Kondoh et al., "The Akt/mTOR pathway assures the synthesis of HIF-1alpha protein in a glucose- and reoxygenation-dependent manner in irradiated tumors," The Journal of Biological Chemistry, vol. 284, no. 8, pp. 5332-5342, 2009.

[60] J. B. Brugarolas, F. Vazquez, A. Reddy, W. R. Sellers, and W. G. Kaelin, "TSC2 regulates VEGF through mTOR-dependent and -independent pathways," Cancer Cell, vol. 4, no. 2, pp. 147-158, 2003.

[61] E. A. Dunlop and A. R. Tee, "Mammalian target of rapamycin complex 1: signalling inputs, substrates and feedback mechanisms," Cellular Signalling, vol. 21, no. 6, pp. 827-835, 2009.

[62] R. Bernardi, I. Guernah, D. Jin et al., "PML inhibits HIFlalpha translation and neoangiogenesis through repression of mTOR," Nature, vol. 442, no. 7104, pp. 779-785, 2006.

[63] S. C. Land and A. R. Tee, "Hypoxia-inducible factor laplha is regulated by the mammalian target of rapamycin (mTOR) via an mTOR signaling motif," The Journal of Biological Chemistry, vol. 282, no. 28, pp. 20534-20543, 2007.

[64] A. Kaidi, A. C. Williams, and C. Paraskeva, "Interaction between alpha-catenin and HIF-1 promotes cellular adaptation to hypoxia," Nature Cell Biology, vol. 9, no. 2, pp. 210217, 2007. 
[65] M. M. Baldewijns, I. J. H. Van Vlodrop, P. B. Vermeulen, P. M. M. B. Soetekouw, M. Van Engeland, and A. P. De Bruïne, "VHL and HIF signalling in renal cell carcinogenesis," The Journal of Pathology, vol. 221, no. 2, pp. 125-138, 2010.

[66] M. Höckel and P. Vaupel, "Biological consequences of tumor hypoxia," Seminars in Oncology, vol. 28, no. 2, supplement 8, pp. 36-41, 2001.

[67] P. Vaupel and L. Harrison, "Tumor hypoxia: causative factors, compensatory mechanisms, and cellular response," Oncologist, vol. 9, supplement 5, pp. 4-9, 2004.

[68] A. Chavez, L. F. Miranda, P. Pichiule, and J. C. Chavez, "Mitochondria and hypoxia-induced gene expression mediated by hypoxia-inducible factors," Annals of the New York Academy of Sciences, vol. 1147, pp. 312-320, 2008.

[69] Y. Jiang, W. Zhang, K. Kondo et al., "Gene expression profiling in a renal cell carcinoma cell line: dissecting VHL and hypoxia-dependent pathways," Molecular Cancer Research, vol. 1, no. 6, pp. 453-462, 2003.

[70] W. G. Kaelin Jr., "The von Hippel-Lindau tumour suppressor protein: $\mathrm{O}_{2}$ sensing and cancer," Nature Reviews Cancer, vol. 8, no. 11, pp. 865-873, 2008.

[71] J. A. Bertout, S. A. Patel, and M. C. Simon, "The impact of $\mathrm{O}_{2}$ availability on human cancer," Nature Reviews Cancer, vol. 8, no. 12, pp. 967-975, 2008.

[72] M. Milani and A. L. Harris, "Targeting tumour hypoxia in breast cancer," European Journal of Cancer, vol. 44, no. 18, pp. 2766-2773, 2008.

[73] J. J. Hung, M. H. Yang, H. S. Hsu, W. H. Hsu, J. S. Liu, and K. $\mathrm{J}$. Wu, "Prognostic significance of hypoxia-inducible factorlalpha, TWIST1 and Snail expression in resectable non-small cell lung cancer," Thorax, vol. 64, no. 12, pp. 1082-1089, 2009.

[74] C. A. Scrideli, C. G. Carlotti, J. F. Mata et al., "Prognostic significance of co-overexpression of the EGFR/IGFBP-2/HIF2A genes in astrocytomas," Journal of Neuro-Oncology, vol. 83, no. 3, pp. 233-239, 2007.

[75] K. M. Comerford, T. J. Wallace, J. Karhausen, N. A. Louis, M. C. Montalto, and S. P. Colgan, "Hypoxia-inducible factor1-dependent regulation of the multidrug resistance (MDR1) gene," Cancer Research, vol. 62, no. 12, pp. 3387-3394, 2002.

[76] S. Park, C. Shimizu, T. Shimoyama et al., "Gene expression profiling of ATP-binding cassette (ABC) transporters as a predictor of the pathologic response to neoadjuvant chemotherapy in breast cancer patients," Breast Cancer Research and Treatment, vol. 99, no. 1, pp. 9-17, 2006.

[77] H. Yasuda, "Solid tumor physiology and hypoxia-induced chemo/radio-resistance: novel strategy for cancer therapy: nitric oxide donor as a therapeutic enhancer," Nitric OxideBiology and Chemistry, vol. 19, no. 2, pp. 205-216, 2008.

[78] A. V. Salnikov, N. E. Heldin, L. B. Stuhr et al., "Inhibition of carcinoma cell-derived VEGF reduces inflammatory characteristics in xenograft carcinoma," International Journal of Cancer, vol. 119, no. 12, pp. 2795-2802, 2006.

[79] Y. Boucher, L. T. Baxter, and R. K. Jain, "Interstitial pressure gradients in tissue-isolated and subcutaneous tumors: implications for therapy," Cancer Research, vol. 50, no. 15, pp. 4478-4484, 1990.

[80] N. Qayum, R. J. Muschel, H. I. Jae et al., "Tumor vascular changes mediated by inhibition of oncogenic signaling," Cancer Research, vol. 69, no. 15, pp. 6347-6354, 2009.

[81] S. Pastorekova, J. Kopacek, and J. Pastorek, "Carbonic anhydrase inhibitors and the management of cancer," Current
Topics in Medicinal Chemistry, vol. 7, no. 9, pp. 865-878, 2007.

[82] S. E. Rademakers, P. N. Span, J. H. A. M. Kaanders, F. C. G. J. Sweep, A. J. Van der Kogel, and J. Bussink, "Molecular aspects of tumour hypoxia," Molecular Oncology, vol. 2, no. 1, pp. 4153, 2008.

[83] N. Raghunand and R. J. Gillies, "pH and chemotherapy," Novartis Foundation Symposium, vol. 240, pp. 199-211, 2001.

[84] L. M. Brown, R. L. Cowen, C. Debray et al., "Reversing hypoxic cell chemoresistance in vitro using genetic and small molecule approaches targeting hypoxia inducible factor-1," Molecular Pharmacology, vol. 69, no. 2, pp. 411-418, 2006.

[85] M. Abend, "Reasons to reconsider the significance of apoptosis for cancer therapy," International Journal of Radiation Biology, vol. 79, no. 12, pp. 927-941, 2003.

[86] D. Eriksson and T. Stigbrand, "Radiation-induced cell death mechanisms," Tumor Biology, vol. 31, no. 4, pp. 363-372, 2010.

[87] M. I. Koukourakis, A. Giatromanolaki, E. Sivridis et al., "Hypoxia-inducible factor (HIF1A and HIF2A), angiogenesis, and chemoradiotherapy outcome of squamous cell headand-neck cancer," International Journal of Radiation Oncology Biology Physics, vol. 53, no. 5, pp. 1192-1202, 2002.

[88] B. J. Moeller, Y. Cao, C. Y. Li, and M. W. Dewhirst, "Radiation activates HIF-1 to regulate vascular radiosensitivity in tumors: role of reoxygenation, free radicals, and stress granules," Cancer Cell, vol. 5, no. 5, pp. 429-441, 2004.

[89] N. Chadderton, R. L. Cowen, F. C. D. Sheppard et al., "Dual responsive promoters to target therapeutic gene expression to radiation-resistant hypoxic tumor cells," International Journal of Radiation Oncology Biology Physics, vol. 62, no. 1, pp. 213-222, 2005.

[90] M. I. Koukourakis, S. M. Bentzen, A. Giatromanolaki et al., "Endogenous markers of two separate hypoxia response pathways (hypoxia inducible factor 2 alpha and carbonic anhydrase 9) are associated with radiotherapy failure in head and neck cancer patients recruited in the CHART randomized trial," Journal of Clinical Oncology, vol. 24, no. 5, pp. 727-735, 2006.

[91] E. Metzen, J. Zhou, W. Jelkmann, J. Fandrey, and B. Brüne, "Nitric oxide impairs normoxic degradation of HIF-1alpha by inhibition of prolyl hydroxylases," Molecular Biology of the Cell, vol. 14, no. 8, pp. 3470-3481, 2003.

[92] L. Milas, K. Mason, N. Hunter et al., "In vivo enhancement of tumor radioresponse by C225 antiepidermal growth factor receptor antibody," Clinical Cancer Research, vol. 6, no. 2, pp. 701-708, 2000.

[93] T. J. Kim, J. W. Lee, S. Y. Song et al., "Increased expression of pAKT is associated with radiation resistance in cervical cancer," British Journal of Cancer, vol. 94, no. 11, pp. 16781682, 2006.

[94] J. A. Bonner, P. M. Harari, J. Giralt et al., "Radiotherapy plus cetuximab for squamous-cell carcinoma of the head and neck," New England Journal of Medicine, vol. 354, no. 6, pp. 567-578, 2006.

[95] R. Prevo, E. Deutsch, O. Sampson et al., "Class I PI3 kinase inhibition by the pyridinylfuranopyrimidine inhibitor PI103 enhances tumor radiosensitivity," Cancer Research, vol. 68, no. 14, pp. 5915-5923, 2008.

[96] J. S. Chen, L. J. Zhou, M. Entin-Meer et al., "Characterization of structurally distinct, isoform-selective phosphoinositide 3'-kinase inhibitors in combination with radiation in the 
treatment of glioblastoma," Molecular Cancer Therapeutics, vol. 7, no. 4, pp. 841-850, 2008.

[97] X. Li, E. A. Kimbrel, D.J. Kenan, and D. P. McDonnell, "Direct interactions between corepressors and coactivators permit the integration of nuclear receptor-mediated repression and activation," Molecular Endocrinology, vol. 16, no. 7, pp. 14821491, 2002.

[98] H. Y. Lin, M. Sun, H. Y. Tang et al., "L-thyroxine vs. 3,5,3'-triiodo-L-thyronine and cell proliferation: activation of mitogen-activated protein kinase and phosphatidylinositol 3-kinase," American Journal of Physiology, vol. 296, no. 5, pp. C980-C991, 2009.

[99] L. C. Moeller, X. Cao, A. M. Dumitrescu, H. Seo, and S. Refetoff, "Thyroid hormone mediated changes in gene expression can be initiated by cytosolic action of the thyroid hormone receptor beta through the phosphatidylinositol 3kinase pathway," Nuclear Receptor Signaling Atlas, vol. 4, p. e020, 2006.

[100] F. Furuya, J. A. Hanover, and S. Y. Cheng, "Activation of phosphatidylinositol 3-kinase signaling by a mutant thyroid hormone $\beta$ receptor," Proceedings of the National Academy of Sciences of the United States of America, vol. 103, no. 6, pp. 1780-1785, 2006.

[101] Y. Hiroi, H. H. Kim, H. Ying et al., "Rapid nongenomic actions of thyroid hormone," Proceedings of the National Academy of Sciences of the United States of America, vol. 103, no. 38, pp. 14104-14109, 2006.

[102] L. A. Palmer, G. L. Semenza, M. H. Stoler, and R. A. Johns, "Hypoxia induces type II NOS gene expression in pulmonary artery endothelial cells via HIF-1," American Journal of Physiology, vol. 274, no. 2, pp. L212-L219, 1998.

[103] R. Liu, Z. Li, S. Bai et al., "Mechanism of cancer cell adaptation to metabolic stress: proteomics identification of a novel thyroid hormone-mediated gastric carcinogenic signaling pathway," Molecular \& Cellular Proteomics, vol. 8, no. 1, pp. 70-85, 2009.

[104] H. Y. Lin, S. Zhang, B. L. West et al., "Identification of the putative MAP kinase docking site in the thyroid hormone receptor-alpha1 DNA-binding domain: functional consequences of mutations at the docking site," Biochemistry, vol. 42, no. 24, pp. 7571-7579, 2003.

[105] Y. Ma, P. Freitag, J Zhou, B. Brüne, S. Frede, and J. Fandrey, "Thyroid hormone induces erythropoietin gene expression through augmented accumulation of hypoxiainducible factor-1," American Journal of Physiology, vol. 287, no. 3, pp. R600-R607, 2004.

[106] T. Otto and J. Fandrey, "Thyroid hormone induces hypoxiainducible factor lalpha gene expression through thyroid hormone receptor beta/retinoid $\mathrm{x}$ receptor alpha-dependent activation of hepatic leukemia factor," Endocrinology, vol. 149, no. 5, pp. 2241-2250, 2008.

[107] W. S. Simonides, M. A. Mulcahey, E. M. Redout et al., "Hypoxia-inducible factor induces local thyroid hormone inactivation during hypoxic-ischemic disease in rats," The Journal of Clinical Investigation, vol. 118, no. 3, pp. 975-983, 2008.

[108] A. M. Jubb, T. Q. Pham, A. M. Hanby et al., "Expression of vascular endothelial growth factor, hypoxia inducible factor lalpha, and carbonic anhydrase IX in human tumours," Journal of Clinical Pathology, vol. 57, no. 5, pp. 504-512, 2004.

[109] D. C. Allred, G. M. Clark, R. Elledge et al., "Association of p53 protein expression with tumor cell proliferation rate and clinical outcome in node-negative breast cancer," Journal of the National Cancer Institute, vol. 85, no. 3, pp. 200-206, 1993.

[110] J. Pouysségur, F. Dayan, and N. M. Mazure, "Hypoxia signalling in cancer and approaches to enforce tumour regression," Nature, vol. 441, no. 7092, pp. 437-443, 2006.

[111] P. Hou, D. Liu, Y. Shan et al., "Genetic alterations and their relationship in the phosphatidylinositol 3-kinase/Akt pathway in thyroid cancer," Clinical Cancer Research, vol. 13, no. 4, pp. 1161-1170, 2007.

[112] Y. Wang, P. Hou, H. Yu et al., "High prevalence and mutual exclusivity of genetic alterations in the phosphatidylinositol3-kinase/Akt pathway in thyroid tumors," Journal of Clinical Endocrinology and Metabolism, vol. 92, no. 6, pp. 2387-2390, 2007.

[113] G. Bunone, P. Vigneri, L. Mariani et al., "Expression of angiogenesis stimulators and inhibitors in human thyroid tumors and correlation with clinical pathological features," American Journal of Pathology, vol. 155, no. 6, pp. 1967-1976, 1999.

[114] A. B. Kilicarslan, M. Ogus, C. Arici, H. E. Pestereli, M. Cakir, and G. Karpuzoglu, "Clinical importance of vascular endothelial growth factor (VEGF) for papillary thyroid carcinomas," APMIS, vol. 111, no. 3, pp. 439-443, 2003.

[115] C. M. Lennard, A. Patel, J. Wilson et al., "Intensity of vascular endothelial growth factor expression is associated with increased risk of recurrence and decreased disease-free survival in papillary thyroid cancer," Surgery, vol. 129, no. 5, pp. 552-558, 2001.

[116] J. M. Vieira, S. C. Santos, C. Espadinha et al., "Expression of vascular endothelial growth factor (VEGF) and its receptors in thyroid carcinomas of follicular origin: a potential autocrine loop," European Journal of Endocrinology, vol. 129, no. 5, pp. 552-558, 2005.

[117] R. T. Kloos, M. H. Shah, M. D. Ringel et al., "Phase II trial of sorafenib in metastatic thyroid cancer," Journal of Clinical Oncology, vol. 27, no. 10, pp. 1675-1684, 2009.

[118] S. A. Wells Jr., J. E. Gosnell, R. F. Gagel et al., "Vandetanib for the treatment of patients with locally advanced or metastatic hereditary medullary thyroid cancer," American Journal of Clinical Oncology, vol. 28, no. 5, pp. 767-772, 2010.

[119] V. Gupta-Abramson, A. B. Troxel, A. Nellore et al., "Phase II trial of sorafenib in advanced thyroid cancer," Journal of Clinical Oncology, vol. 26, no. 29, pp. 4714-4719, 2008.

[120] M. B. Bass, S. I. Sherman, M. J. Schlumberger et al., "Biomarkers as predictors of response to treatment with motesanib in patients with progressive advanced thyroid cancer," The Journal of Clinical Endocrinology \& Metabolism, vol. 95, no. 11, pp. 5018-5027, 2010.

[121] J. T. Erler, K. L. Bennewith, M. Nicolau et al., "Lysyl oxidase is essential for hypoxia-induced metastasis," Nature, vol. 440, no. 7088, pp. 1222-1226, 2006.

[122] X. Y. Zhao, T. T. Chen, and L. Xia, "Hypoxia inducible factor1 mediates expression of galectin-1: the potential role in migration/invasion of colorectal cancer cells," Carcinogenesis, vol. 31, no. 8, pp. 1367-1375, 2010.

[123] J. Chen, N. Imanaka, J. Chen, and J. D. Griffin, "Hypoxia potentiates Notch signaling in breast cancer leading to decreased E-cadherin expression and increased cell migration and invasion," British Journal of Cancer, vol. 102, no. 2, pp. 351-360, 2010.

[124] S. Scarpino, A. Stoppacciaro, F. Ballerini et al., "Papillary carcinoma of the thyroid: hepatocyte growth factor (HGF) 
stimulates tumor cells to release chemokines active in recruiting dendritic cells," American Journal of Pathology, vol. 156, no. 3, pp. 831-837, 2000.

[125] S. Scarpino, F. C. D’Alena, A. Di Napoli, F. Ballarini, M. Prat, and L. P. Ruco, "Papillary carcinoma of the thyroid: evidence for a role for hepatocyte growth factor (HGF) in promoting tumour angiogenesis," The Journal of Pathology, vol. 199, no. 2, pp. 243-250, 2003.

[126] T. Brabletz, A Jung, S. Reu et al., "Variable beta-catenin expression in colorectal cancers indicates tumor progression driven by the tumor environment," Proceedings of the National Academy of Sciences of the United States of America, vol. 98, no. 18, pp. 10356-10361, 2001.

[127] S. Scarpino, F. C. d'Alena, A. Di Napoli, A. Pasquini, A. Marzullo, and L. P. Ruco, "Increased expression of Met protein is associated with up-regulation of hypoxia inducible factor-I (HIF-I) in tumour cells in papillary carcinoma of the thyroid," The Journal of Pathology, vol. 202, no. 3, pp. 352358,2004

[128] B. J. Moeller and M. W. Dewhirst, "HIF-1 and tumour radiosensitivity," British Journal of Cancer, vol. 95, no. 1, pp. $1-5,2006$.

[129] G. L. Semenza, "Defining the role of hypoxia-inducible factor 1 in cancer biology and therapeutics," Oncogene, vol. 29, no. 5, pp. 625-634, 2010.

[130] C. Magnon, P. Opolon, M. Ricard et al., "Radiation and inhibition of angiogenesis by canstatin synergize to induce HIF- $1 \alpha$-mediated tumor apoptotic switch," The Journal of Clinical Investigation, vol. 117, no. 7, pp. 1844-1855, 2007. 


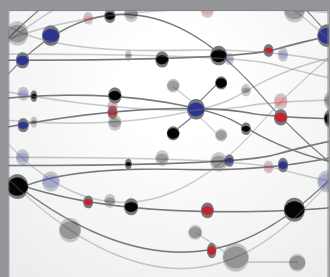

The Scientific World Journal
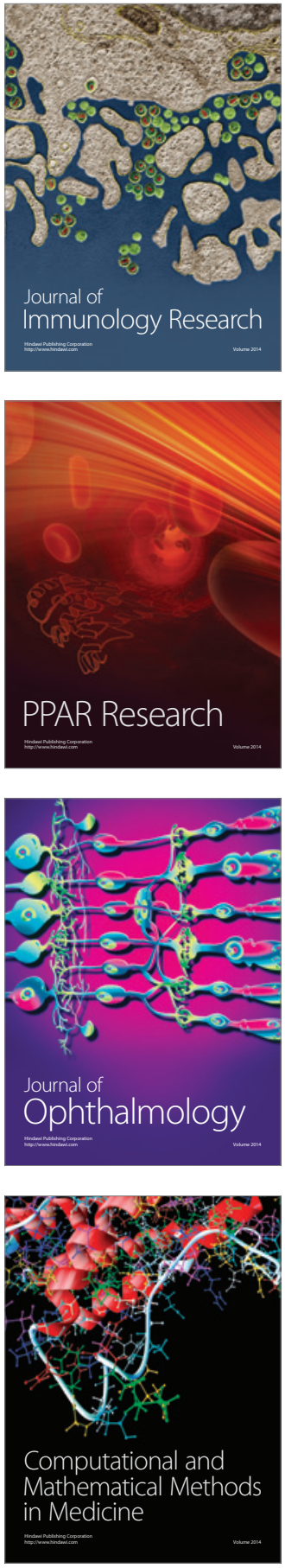

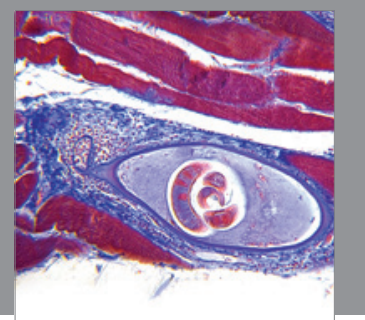

Gastroenterology

Research and Practice
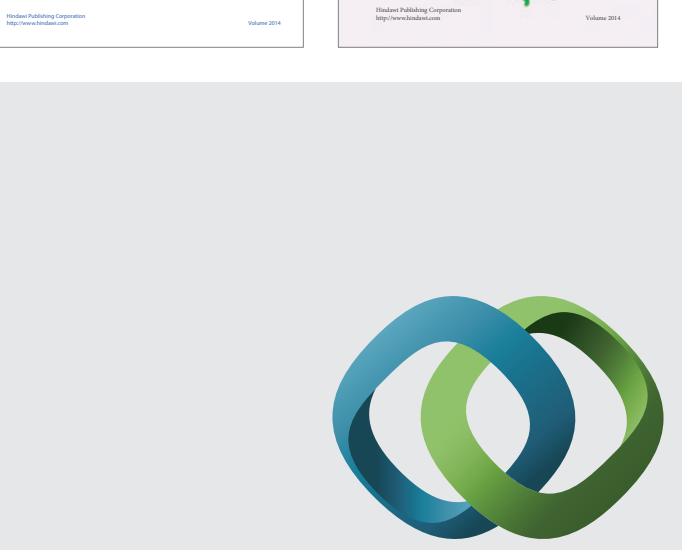

\section{Hindawi}

Submit your manuscripts at

http://www.hindawi.com
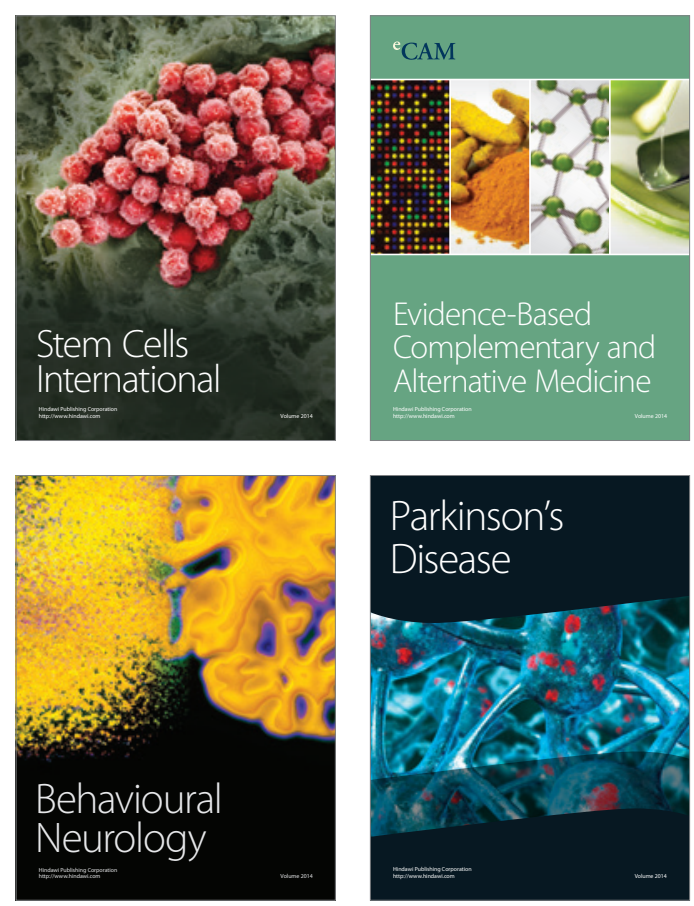

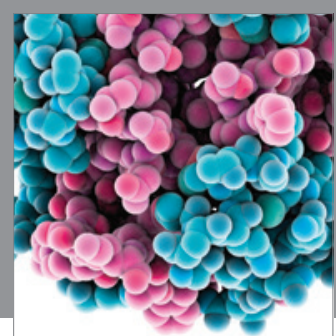

Journal of
Diabetes Research

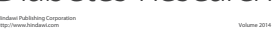

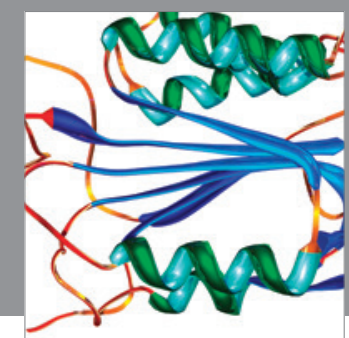

Disease Markers
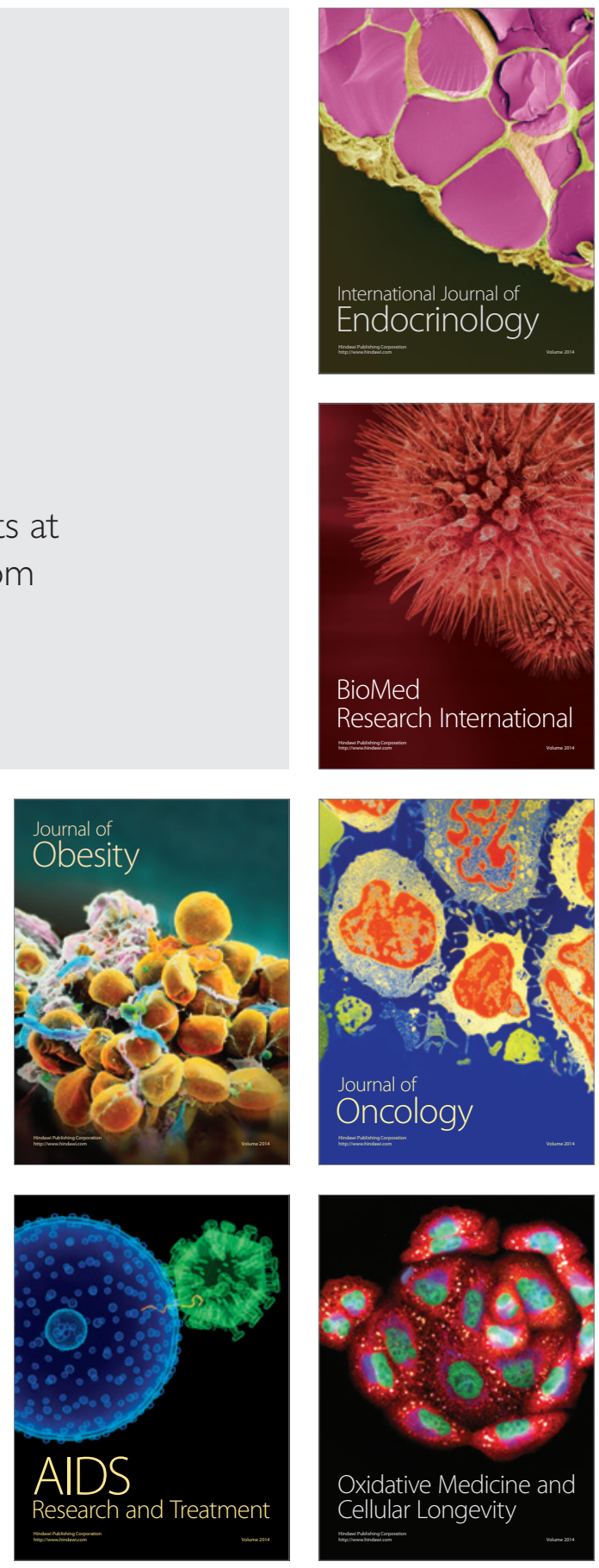